This document is the accepted manuscript version of the following article:

Authors: M. Fraldi, S. Palumbo, A.R. Carotenuto, A. Cutolo, N. Pugno

Title: Generalized multiple peeling theory uploading hyperelasticity and pre-stress

Journal: Extreme Mechanics Letters

Publisher doi: 10.1016/j.eml.2020.101085

This manuscript version is made available under the CC-BY-NC-ND 4.0 license Originally uploaded to URL: http://www.ing.unitn.it/ pugno/NP_PDF/

PostPrint/2020-Fraldi-peeling.pdf on 26/11/2020 


\title{
Generalized multiple peeling theory uploading hyperelasticity and pre-stress
}

\author{
M. Fraldi*1,2 ${ }^{*}$ S. Palumbo ${ }^{\dagger 1}$, A.R. Carotenuto ${ }^{\ddagger 1}$, A. Cutolo ${ }^{\S 1}$, and N. Pugno ${ }^{\llbracket(13,4}$ \\ 1 Department of Structures for Engineering and Architecture, University of Napoli Federico II, Italy \\ ${ }^{2}$ Institute for Applied Sciences and Intelligent Systems, National Research Council of Italy, Italy \\ ${ }^{3}$ Laboratory of Bio-inspired, Bionic, Nano, Meta Materials and Mechanics, Department of Civil, Environmental and Mechanical Engineering, \\ University of Trento, Italy \\ ${ }^{4}$ School of Engineering and Materials Science, Queen Mary University of London, London, UK
}

September 22, 2020

\begin{abstract}
Extreme adhesion mechanisms are shown in a wide number of situations in nature, from structures of single cells and spider web anchorages to hierarchical organization of ends and pads of insects and reptiles. With this in mind, we here propose a general formulation of multiple peeling theory accounting for geometrical and constitutive nonlinearities, i.e. large deformations and hyperelasticity. Pre-stress of adherent tracts and different initial V-shaped configurations of the tapes have been also included in the modelling. By following an analytical approach, closed-form solutions and explicit formulas were given for predicting pull-off critical forces and optimality conditions maximizing the adhesion strength, as well as for tracing the evolution of the delamination process, from the detaching onset to its progressive behaviour. Kendall and previous linear multiple peeling theory approaches were all recovered as limit cases, gaining insights into the role played by nonlinearities and pre-stress and also demonstrating -with mathematically rigorous arguments- the asymptotic character of the detaching phenomenon, to date only glimpsed from experimental evidences. Example applications and simple key tests were provided to show the effectiveness of the approach, which is felt could help to interpret peeling in nature and to design materials with enhanced adhesive properties.
\end{abstract}

\section{Introduction}

Adhesion plays a crucial role in nature across the scales for optimizing locomotion and climbing of insects, arachnids and reptiles [1], as predating strategy in anchorage systems of spider webs and for activating mechanotransduction signals prodromal to migration and mitosis in adherent cells (see figure 1). In most cases, enhanced adhesion strength seems to be in fact achieved by means of hairy

\footnotetext{
*fraldi@unina.it

†stefania.palumbo@unina.it

† angelorosario.carotenuto@unina.it

§arsenio.cutolo@unina.it

In nicola.pugno@unitn.it (corresponding author)
} 
and hierarchical arrangements of the structures deputed to attachment [2-10]. As an example, gecko and insects anchorages are organized as splitting tape-like terminal elements at different length scales, namely from the macroscopic level of legs and digits up to the micro- and nano-scales of setae and spatular tips $[3-5,7,11,12]$, the latter directly interacting with the contact substrate by establishing van der Waals forces $[2,13]$. As a result, the observation of these biological structures and the recognition of their potentialities have inspired the conception of novel adhesive devices and micropatterned surfaces in which optimized biomimetic attachment/detachment mechanisms might be employed for industrial, robotic as well as biomedical applications [14-21]. Also, bonding/debonding models could be helpfully used to characterize the mechanics of adherent living cells and their interaction with the external environment through focal adhesions at the microscopic scale [22-24], as well as, at the macroscopic level, the dissection and rupture events potentially occurring in vascular tissue layers $[25,26]$.

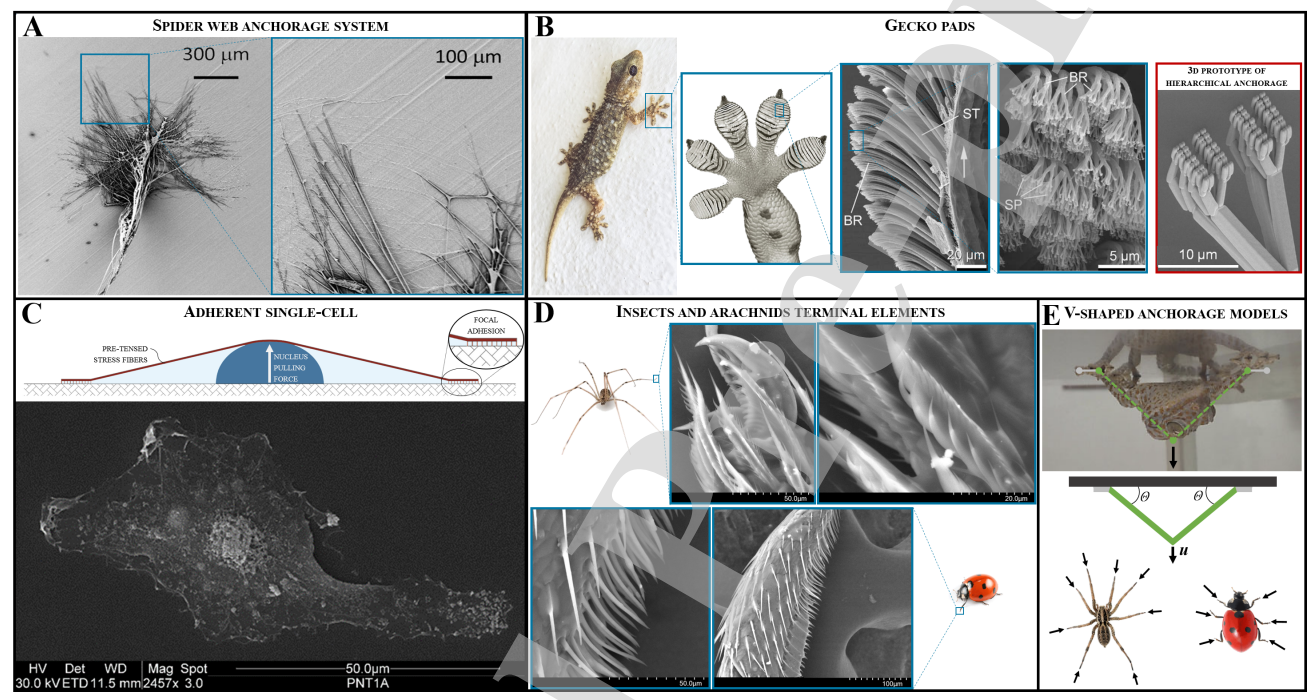

Figure 1: A) Scanning electron microscope image of a spider web anchorage system. Multiple and hierarchical organization of tape-like adhesive elements is highlighted. B) Gecko with pads allowing extreme adhesion for climbing surfaces by virtue of the hierarchical arrangement of its anchoring structures, reported in the scanning electron micrographs adapted from the work by Gao et al. [11] (therein, ST: seta, SP: spatula, BR: branch). On the right, a prototype of the multiple anchorage scheme of the setae showing three levels of branching, reported from the work by Tricinci et al. [27]. C) Scheme and scanning electron microscope image of a single-cell -specifically, a normal adult prostatic epithelial cell PNT1A (ECACC 95012614)- adhering to a flat substrate. The tensed stress fibers of the elongated cell anchor to the substrate by establishing focal adhesion sites, whose adhesion strength has to prevent the cell detachment that the pulling force due to the reacting compressed nucleus would induce. D) Scanning electron microscope images of insects and arachnids terminal ends evidencing the presence of pulvilli, essential for adhesion. E) Schematization of gecko (whose picture is adapted from the work by Pugno [28]), spider and lady bug during ceiling situation through a simplified macroscopic multiple anchorage model.

In this direction, understanding the mechanics at the basis of peeling mechanisms that occur at the adhesive interface of film-substrate systems has found wide interest in the scientific literature of the last decades. In fact, starting from seminal works such as [29-32], several theoretical and experimental studies have been then conducted to the aim of investigating the de-adhesion process of tape-like (or spatula-like) elements $[5,11,22,28,33-40]$. These have alternatively taken into account the role played by pre-tension $[34,38,39]$, bending stiffness [41-43], large deformations $[44,45]$ and multiple or hi- 
erarchical arrangements of the strips [12,28,33,35], as well as the influence of the roughness [46,47] or of the compliance [36] of the underlying substrate, on the peeling.

Within this framework, Kendall single peeling model [29], in which an energy-based approach is adopted for studying peeling of a thin adhesive film from a rigid substrate, has been recently extended by Pugno [28] to implement a multiple peeling theory, which appears as necessary for more faithfully describing and explaining the mechanics of both natural and bio-inspired complex anchorage structures. Specifically, in [28], detaching of a system of linear elastic films, in part adhering over a flat rigid substrate, has been modelled, by then focusing the attention on the delamination of $\mathrm{V}$-shaped schemes and of micro-structured tapes apt to characterize gecko setae and insect specializations used to optimally hang on surfaces. On these bases, double peeling of elastic endless tapes and axisymmetric detachment of circular membranes have been for example analytically studied in [22], while a numerical model has been proposed in [35] to analyze double peeling of symmetrical/asymmetrical strips configurations under different loading conditions and to model spider web-inspired multiple anchorages. Good agreement between results provided by double peeling experiments and the outcomes from mechanical models grounded on the multiple peeling theory has been also shown in [37, 39], this contributing to corroborate the validity of that proposed approach. In particular, influence of prestress was explored in [39], while an experiment-guided piece-wise linear description of the strips was introduced in [37]. Furthermore, the peeling progression of periodic V-shaped arrangements of adhesive elements interacting with a compliant substrate has been modelled [36], by removing the hypothesis of rigid medium, which can be considered however realistic for a large class of physical applications. Finally, in a very recent work [48], optimization of the detachment process of multiple anchorage systems has been investigated by combining the mechanisms of elastic deformation, interface delamination and fracture of the tapes.

Within this framework, the present work deals with the formulation of a hyperelastic theory of multiple peeling, by taking into account large deformations and nonlinear behaviour of the tapes, as well as the effects of a pre-stretch possibly stored both in their adherent and free (i.e. non-adhesive) tracts. To this aim, after establishing the general equations governing the peeling process in the case of arbitrary hyperelastic constitutive law and arrangement of the tapes, which also leads to an extension of the well-known Kendall equation [29], a first example of symmetrical multiple peeling of linear elastic elements adhering on a rigid ground is analytically studied. Then, the critical (i.e. the first) pull-off event and the quasi-static detachment process of a V-shaped system made of two (incompressible) neo-Hookean strips, induced by symmetrically applied loads, are modelled. By following this way, pulling forces and peeling angles are derived as functions of the pre-stretches and of the geometrical, constitutive and adhesive properties of the system, and optimal (i.e. maximum) adhesion configurations, limit (asymptotic) behaviours, discrepancies between linear and nonlinear models and potential occurrence of asymmetrical detachment are in particular investigated. Finally, some comparisons with results obtained by preliminary experimental tests are also shown.

\section{Multiple peeling of hyperelastic adhesive tapes}

Let us consider the scheme of multiple anchorage illustrated in figure $2 \mathrm{~A}$, in which $n$ adhesive strips partially adhere over a flat (horizontally positioned) rigid substrate and can undergo peeling due to a pulling external displacement prescribed at the hinge-like point to which their non-adhesive extremities converge. Then, with reference to the generic $i$-th strip element, with $i=1, \ldots, n$, the following 
geometrical relations can be written:

$$
\begin{aligned}
& l_{i e}=l_{i e}(u, \alpha)=\sqrt{\left(L_{i} \cos \Theta_{i}+\operatorname{sgn}\left[\left(\hat{\mathbf{L}}_{i} \cdot \hat{\mathbf{e}}_{x}\right)\left(\hat{\boldsymbol{u}} \cdot \hat{\mathbf{e}}_{x}\right)\right] u \cos \alpha\right)^{2}+\left(L_{i} \sin \Theta_{i}+u \sin \alpha\right)^{2}}, \\
& l_{i d}=l_{i d}\left(u, \alpha, d_{i}\right)=\sqrt{\left(L_{i} \cos \Theta_{i}+d_{i}+\operatorname{sgn}\left[\left(\hat{\mathbf{L}}_{i} \cdot \hat{\mathbf{e}}_{x}\right)\left(\hat{\boldsymbol{u}} \cdot \hat{\mathbf{e}}_{x}\right)\right] u \cos \alpha\right)^{2}+\left(L_{i} \sin \Theta_{i}+u \sin \alpha\right)^{2}}, \\
& L_{i d}=L_{i d}\left(d_{i}\right)=L_{i}+d_{i} \Lambda_{i}^{-1}, \\
& \lambda_{i e}=\lambda_{i e}(u, \alpha)=l_{i e} L_{i}^{-1} \\
& \lambda_{i d}=\lambda_{i d}\left(u, \alpha, d_{i}\right)=l_{i d} L_{i d}^{-1}, \\
& \theta_{i e}=\theta_{i e}(u, \alpha)=\arctan \frac{L_{i} \sin \Theta_{i}+u \sin \alpha}{L_{i} \cos \Theta_{i}+\operatorname{sgn}\left[\left(\hat{\mathbf{L}}_{i} \cdot \hat{\mathbf{e}}_{x}\right)\left(\hat{\boldsymbol{u}} \cdot \hat{\mathbf{e}}_{x}\right)\right] u \cos \alpha}, \\
& \theta_{i d}=\theta_{i d}\left(u, \alpha, d_{i}\right)=\arctan \frac{L_{i} \sin \Theta_{i}+u \sin \alpha}{d_{i}+L_{i} \cos \Theta_{i}+\operatorname{sgn}\left[\left(\hat{\mathbf{L}}_{i} \cdot \hat{\mathbf{e}}_{x}\right)\left(\hat{\boldsymbol{u}} \cdot \hat{\mathbf{e}}_{x}\right)\right] u \cos \alpha} .
\end{aligned}
$$

Herein, $u$ and $\alpha$ are respectively the magnitude of the applied displacement $u$ and the angle that it forms with the horizontal direction, $l_{i e}$ and $l_{i d}$ are, in the order, the actual (deformed) strip lengths in case of purely elastic deformation (namely, before the delamination occurs) and of elastic deformation of a strip element detached for a tract $d_{i}$, while $L_{i}$ and $L_{i d}$ are the corresponding initial (undeformed) lengths to be referred to for calculating the actual axial stretches $\lambda_{i e}$ and $\lambda_{i d}$ in the two cases, respectively. In addition, $\Theta_{i} \in[0, \pi / 2]$ identifies the angle that the $i$-th tape originally forms with the rigid support to which it adheres while $\theta_{i e}$ and $\theta_{i d}$ refer to the actual angles that the same strip describes in the purely deformed or also detached configuration. Finally, $\Lambda_{i} \geqslant 1$ is the pre-stretch initially stored by the strip's adherent part, $\hat{\mathbf{L}}_{i}$ and $\hat{\boldsymbol{u}}$ are the unit vectors respectively identifying the reference directions of the $i$-th tape and of the applied displacement in a Cartesian system $\left(\hat{\mathbf{e}}_{x}, \hat{\mathbf{e}}_{y}\right), \operatorname{sgn}[\bullet]$ being the signum function providing the sign of its argument.

By modelling each tape as a hyperelastic isotropic and homogeneous material, its constitutive equation can be generally given as

$$
\Psi_{i}=\Psi_{i}\left(\lambda_{i 1}, \lambda_{i 2}, \lambda_{i 3}\right), \quad \forall i=1, \ldots, n
$$

where the strain-energy density $\Psi_{i}$ is assigned as a function of the principal stretches $\lambda_{i j}, j=1,2,3$, in a way that the principal first Piola-Kirchhoff stresses $P_{i j}=P_{i j}\left(\lambda_{i 1}, \lambda_{i 2}, \lambda_{i 3}\right)$ can be derived as

$$
P_{i j}=\frac{\partial \Psi_{i}}{\partial \lambda_{i j}}, \quad \forall i=1, \ldots, n .
$$

Under the hypothesis that the strip is elastically stretched by $\lambda_{i 1}=\lambda_{i}$ along its longitudinal direction and by imposing that the associated stress regime is uniform and mono-axial along the strip axis, i.e. $P_{i 2}=P_{i 3}=0$, standard arguments of nonlinear elasticity allow to find the transversal (principal) stretches as a function of the longitudinal one, namely $\lambda_{i 2}=\lambda_{i 3}=\lambda_{i T}=\lambda_{i T}\left(\lambda_{i}\right)$. This implies that the free energy density in (2.2) can be given in terms of the sole axial stretch $\lambda_{i}$, say $\hat{\Psi}_{i}=\hat{\Psi}_{i}\left(\lambda_{i}\right)=$ $\left.\Psi_{i}\right|_{\lambda_{i 1}=\lambda_{i 2}=\lambda_{i T}}$. Accordingly, the only not vanishing (longitudinal) nominal stress component can be written as $\hat{P}_{i}=\hat{P}_{i}\left(\lambda_{i}\right)=\partial \hat{\Psi}_{i} / \partial \lambda_{i}$, while the elastic energy stored by the strip as an effect of the deformation can be computed as

$$
\Pi_{i}=\Pi_{i}\left(\lambda_{i}\right)=\int_{\Omega_{i}} \hat{\Psi}_{i}\left(\lambda_{i}\right) d \Omega=V_{i} \hat{\Psi}_{i}\left(\lambda_{i}\right), \quad \forall i=1, \ldots, n
$$

thanks to the uniformity of the stretch over the reference volume of the underformed strip $\Omega_{i}$ and therein being $V_{i}=\operatorname{Vol}\left(\Omega_{i}\right)$. In particular, by denoting with $b_{i}$ and $t_{i}$ the nominal width and the nominal thickness of the $i$-th tape, respectively, $V_{i}$ is provided by

$$
V_{i e}=b_{i} t_{i} L_{i}
$$




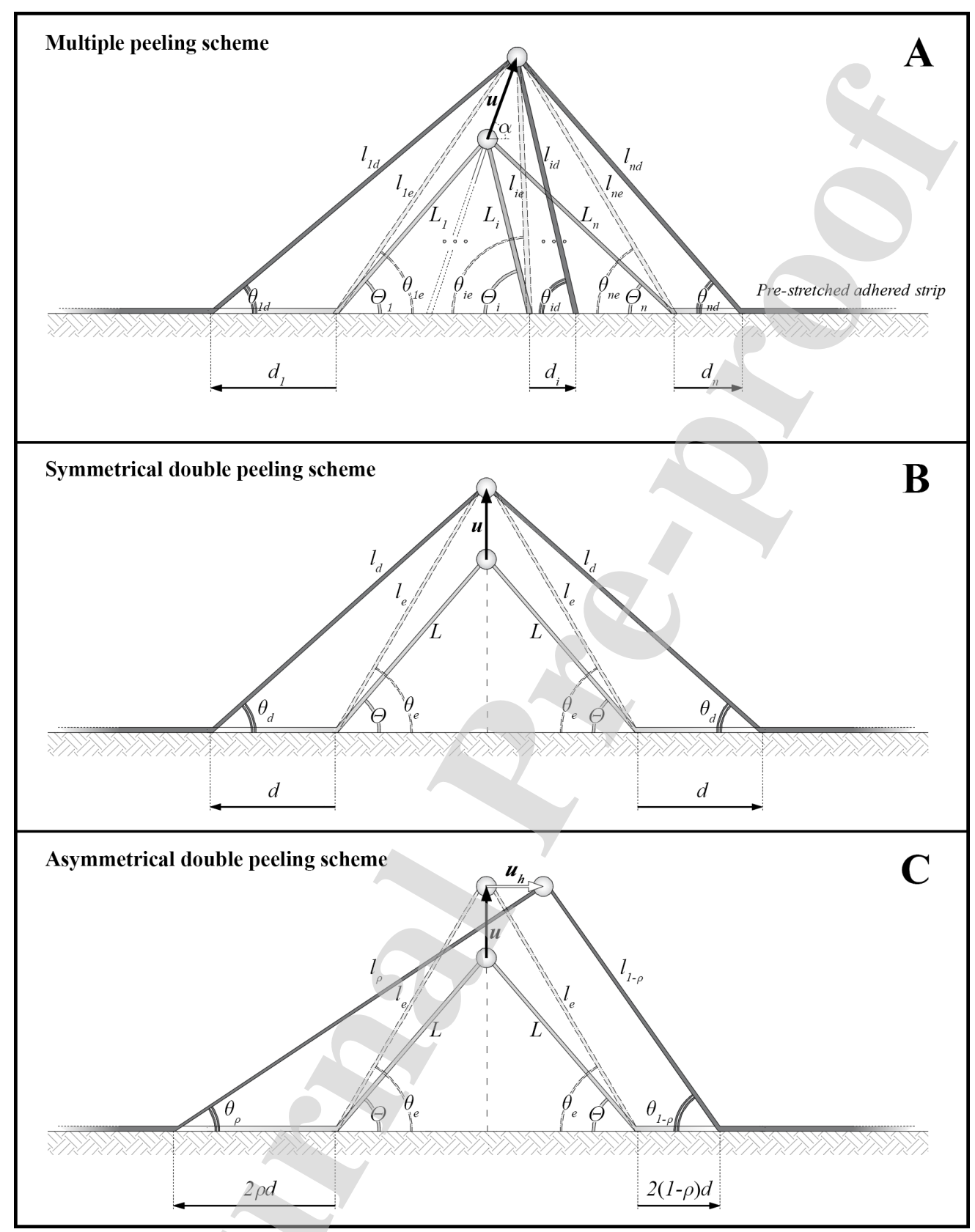

Figure 2: A) Scheme of multiple peeling of a general $n$-tape anchorage system under prescribed displacement. B) Symmetrical double peeling of a V-shaped system under prescribed vertical displacement. C) Asymmetrical double peeling of a V-shaped system under prescribed vertical displacement.

if the element is only elastically deformed, while it coincides with

$$
V_{i d}=V_{i d}\left(d_{i}\right)=b_{i} t_{i} L_{i d}
$$


in the case in which the element is detached for a segment $d_{i}, b_{i} t_{i}$ representing its nominal crosssectional area and $L_{i d}$ being given in (2.1) as a function of $d_{i}$. Therefore, with reference to the two possible states of the generic $i$-th strip, the related elastic energies are respectively provided by

$$
\Pi_{i e}=\Pi_{i e}(u, \alpha)=V_{i e} \Psi_{i e}, \quad \Psi_{i e}=\Psi_{i e}(u, \alpha)=\left.\hat{\Psi}_{i}\left(\lambda_{i}\right)\right|_{\lambda_{i}=\lambda_{i e}}
$$

and by

$$
\Pi_{i d}=\Pi_{i d}\left(u, \alpha, d_{i}\right)=V_{i d} \Psi_{i d}, \quad \Psi_{i d}=\Psi_{i d}\left(u, \alpha, d_{i}\right)=\left.\hat{\Psi}_{i}\left(\lambda_{i}\right)\right|_{\lambda_{i}=\lambda_{i d}},
$$

with $\lambda_{i e}$ and $\lambda_{i d}$ given in (2.1).

Without loss of generality, by neglecting any possibility of interaction (e.g. physical superposition) of the strips' adhering tracts and by following the Griffith criterion $[28,49]$, under the hypothesis of quasi-static process, the detachment of each tape is ruled by the condition

$$
-\frac{\partial}{\partial d_{i}}\left(\sum_{k=1}^{n} \Pi_{k d}\right)=b_{i} R_{i}
$$

$R_{i}$ being the adhesive energy per unit area in a way that $b_{i} R_{i}$ is the dissipative energy contribution required for the creation of a new free surface having width $b_{i}$ and unit length. However, since the elastic energy contribution of the $i$-th strip does not depend on the delamination of the other elements of the anchorage scheme, i.e. on $d_{k}$ with $k \neq i$, equation (2.9) simply results in

$$
-\frac{\partial \Pi_{i d}}{\partial d_{i}}=b_{i} R_{i}
$$

which, by adopting the definition of $\Pi_{i d}$ given in (2.8), after an in-cascade application of derivatives' rules and a some algebraic manipulations, can be explicitly reformulated as follows:

$$
\frac{\Psi_{i d}}{\Lambda_{i}}+P_{i d}\left(\frac{L_{i} \cos \Theta_{i}+\operatorname{sgn}\left[\left(\hat{\mathbf{L}}_{i} \cdot \hat{\mathbf{e}}_{x}\right)\left(\hat{\boldsymbol{u}} \cdot \hat{\mathbf{e}}_{x}\right)\right] u \cos \alpha+d_{i}}{l_{i d}}-\frac{\lambda_{i d}}{\Lambda_{i}}\right)+\frac{R_{i}}{t_{i}}=0,
$$

where $P_{i d}=P_{i d}\left(u, \alpha, d_{i}\right)=\left.\hat{P}_{i}\right|_{\lambda_{i}=\lambda_{i d}}$, with $\lambda_{i d}$ written as in (2.1). Hence, by solving equation (2.11) for all $i=1, \ldots, n$, the quasi-static evolution of the peeling process of each strip can be derived independently from the others, starting from its first detachment. Indeed, once fixed the geometrical and constitutive properties of the $i$-th tape, the previous equation would provide the relationship between the actual detached portion of the tape $d_{i}$ and the applied displacement $\mathbf{u}$, say $\tilde{d}_{i}=\tilde{d}_{i}(u, \alpha)$, for any $u \geq u_{i c r}$, where $u_{i c r}=u_{i c r}(\alpha)$ is the critical value of the displacement magnitude for which peeling of the element initiates. Specifically, this depends on the pulling direction $\alpha$ and is in general derivable as solution of the equation $\tilde{d}_{i}=0$ or, equivalently, of the equation (2.10), hence of the (2.11), particularized for $d_{i}=0$, that is:

$-\left.\frac{\partial \Pi_{i d}}{\partial d_{i}}\right|_{d_{i}=0}=b_{i} R_{i} \Leftrightarrow \frac{\Psi_{i e}}{\Lambda_{i}}+P_{i e}\left(\frac{L_{i} \cos \Theta_{i}+\operatorname{sgn}\left[\left(\hat{\mathbf{L}}_{i} \cdot \hat{\mathbf{e}}_{x}\right)\left(\hat{\boldsymbol{u}} \cdot \hat{\mathbf{e}}_{x}\right)\right] u \cos \alpha}{l_{i e}}-\frac{\lambda_{i e}}{\Lambda_{i}}\right)+\frac{R_{i}}{t_{i}}=0$,

where $P_{i e}=P_{i e}(u, \alpha)=\left.\hat{P}_{i}\right|_{\lambda_{i}=\lambda_{i e}}, \lambda_{i e}$ being instead given in (2.1). This implies that, by solving equation $(2.12)_{2}$ in terms of $u$ for all $i=1, \ldots, n$, one can determine the critical values of the prescribed displacement triggering the delamination of all the $n$ tapes and then, by sorting them from the lowest to the highest one, establish the sequence in which the detachments start as the magnitude of the applied displacement increases, for a fixed inclination.

By proceeding in this way, once found the solutions $\tilde{d}_{i}$ and the corresponding $u_{i c r}$ for $i=1, \ldots, n$, one can derive the total pull-off force to be exerted for imposing the displacement magnitude $u$ along the direction identified by the angle $\alpha$ as:

$$
\tilde{F}=\tilde{F}(u, \alpha)=\sum_{i=1}^{n} \operatorname{sgn}\left[\left(\hat{\mathbf{L}}_{i} \cdot \hat{\mathbf{e}}_{x}\right)\left(\hat{\boldsymbol{u}} \cdot \hat{\mathbf{e}}_{x}\right)\right] \tilde{F}_{i} \cos \left(\alpha-\tilde{\theta}_{i}\right),
$$


where

$$
\tilde{F}_{i}=\tilde{F}_{i}(u, \alpha)=b_{i} t_{i}\left[P_{i e}\left(H\left(u_{i c r}-u\right)-\frac{1}{2} \delta\left(u_{i c r}-u\right)\right)+\tilde{P}_{i d}\left(H\left(u-u_{i c r}\right)-\frac{1}{2} \delta\left(u_{i c r}-u\right)\right)\right]
$$

is the axial force arising within each tape, while

$$
\tilde{\theta}_{i}=\tilde{\theta}_{i}(u, \alpha)=\theta_{i e}\left(H\left(u_{i c r}-u\right)-\frac{1}{2} \delta\left(u_{i c r}-u\right)\right)+\tilde{\theta}_{i d}\left(H\left(u-u_{i c r}\right)-\frac{1}{2} \delta\left(u_{i c r}-u\right)\right)
$$

is the current angle described by the same tape with respect to the horizontal direction. In particular, therein, $\tilde{P}_{i d}=\tilde{P}_{i d}(u, \alpha)=\left.P_{i d}\right|_{d_{i}=\tilde{d}_{i}}$ and $\tilde{\theta}_{i d}=\tilde{\theta}_{i d}(u, \alpha)=\left.\theta_{i d}\right|_{d_{i}=\tilde{d}_{i}}$, while $H(\bullet)$ is the Heaviside step function assuming unit value when its argument is greater than or equal to 0 and null otherwise and $\delta(\bullet)$ is the Dirac delta function assuming unit value when its argument is equal to 0 and null otherwise. Then, the adhesion strength offered by the overall anchorage system, defined as the force to be applied in the direction of the displacement in order to induce the first detachment of (at least) one tape, can be calculated as

$$
F_{c r}=F_{c r}(\alpha)=\left.\tilde{F}\right|_{u=u_{c r}}=\sum_{i=1}^{n} \operatorname{sgn}\left[\left(\hat{\mathbf{L}}_{i} \cdot \hat{\mathbf{e}}_{x}\right)\left(\hat{\boldsymbol{u}} \cdot \hat{\mathbf{e}}_{x}\right)\right] b_{i} t_{i}\left[\left.P_{i e}\right|_{u=u_{c r}} \cos \left(\alpha-\left.\theta_{i e}\right|_{u=u_{c r}}\right)\right],
$$

herein being $u_{c r}=u_{c r}(\alpha)=\min _{i} u_{i c r}$ the lowest critical displacement associated to the overall system.

Therefore, one can finally envisage the following general evolution of the multiple anchorage scheme: by imposing a growing pulling displacement to the common vertex of the strips, they all respond through a purely elastic deformation, without detaching, until the application of the critical load $u_{c r}$; this then activates the delamination process of at least one tape, whereupon all the tapes progressively start to detach by producing a slope change in the total pull-off force $\tilde{F}$ versus displacement curve.

On the other hand, it is worth noting that equation (2.11), written for the $i$-th tape with respect to the initial (undeformed) configuration of the system, can be properly reformulated with reference to its current (deformed) configuration. Indeed, by taking into account that the following geometrical relationship holds true (see figure $2 \mathrm{~A}$ ):

$$
L_{i} \cos \Theta_{i}+\operatorname{sgn}\left[\left(\hat{\mathbf{L}}_{i} \cdot \hat{\mathbf{e}}_{x}\right)\left(\hat{\boldsymbol{u}} \cdot \hat{\mathbf{e}}_{x}\right)\right] u \cos \alpha+d_{i}=l_{i d} \cos \theta_{i d},
$$

by substituting it into $(2.11)$, one obtains

$$
\frac{\hat{\Psi}_{i}\left(\lambda_{i d}^{\prime}\right)}{\Lambda_{i}}+\hat{P}_{i}\left(\lambda_{i d}^{\prime}\right)\left(\cos \theta_{i d}^{\prime}-\frac{\lambda_{i d}^{\prime}}{\Lambda_{i}}\right)+\frac{R_{i}}{t_{i}}=0
$$

which relates the current axial stretch $\lambda_{i d}^{\prime}$ arising within the $i$-th tape when detached for a tract $d_{i}$ and the current angle $\theta_{i d}^{\prime}$ that it forms with the horizontal direction, the two quantities being therein regarded as independent variables. As a matter of fact, since such equation refers to the current configuration and loses any dependence on $d_{i}$, it both governs the critical condition of first detachment of the tape and the progression of its delamination. Therefore, its solution would in principle provide the critical axial stretch - and thus, indirectly, the related critical force- required to act on the $i$-th tape for initiating detachment, as a function of the critical peeling angle, as well as the same relation would provide the current element's stretch -whence the current force- as a function of the current inclination during the delamination process evolution. In this latter case, however, one would miss the control of the initial arrangement of the system (e.g. on $\Theta_{i}$ ) and of the magnitude and orientation of the pulling displacement.

Finally, it can be highlighted that equation (2.18) de facto represents a generalization of the wellknown Kendall equation [29] (which rules the single peeling of a linear elastic tape from a rigid 
substrate at a constant angle) to the case of hyperelastic elements in presence of a pre-stretch within the adherent tracts. Indeed, it is known from continuum mechanics [50] that, by considering a homogeneous and isotropic linear elastic strip axially stretched by $\lambda_{i}$ and undergoing mono-axial stress regime, its strain energy function and longitudinal nominal stress component are provided by

$$
\hat{\Psi}_{i}^{l i n}\left(\lambda_{i}\right)=\frac{1}{2} Y_{i}\left(\lambda_{i}-1\right)^{2}, \quad \hat{P}_{i}^{l i n}\left(\lambda_{i}\right)=Y_{i}\left(\lambda_{i}-1\right),
$$

respectively, $Y_{i}$ being the strip's Young modulus. Therefore, by assuming $\hat{\Psi}_{i}=\hat{\Psi}_{i}^{l i n}$ and $\hat{P}_{i}=\hat{P}_{i}^{l i n}$, equation (2.18) provides

$$
\frac{Y_{i}}{2 \Lambda_{i}}\left(\lambda_{i d}^{\prime}-1\right)^{2}+Y_{i}\left(\lambda_{i d}^{\prime}-1\right)\left(\cos \theta_{i d}^{\prime}-\frac{\lambda_{i d}^{\prime}}{\Lambda_{i}}\right)+\frac{R_{i}}{t_{i}}=0
$$

which, reformulated in terms of strip's axial force, say $F_{i d}^{\prime}$, by exploiting the constitutive relation $\lambda_{i d}^{\prime}=1+F_{i d}^{\prime} /\left(b_{i} t_{i} Y_{i}\right)$, gives

$$
\left(\frac{F_{i d}^{\prime}}{b_{i}}\right)^{2} \frac{1}{2 t_{i} Y_{i} \Lambda_{i}}+\frac{F_{i d}^{\prime}}{b_{i}}\left(\frac{1}{\Lambda_{i}}-\cos \theta_{i d}^{\prime}\right)-R_{i}=0
$$

In fact, this equation, which coincides with the Kendall's one [29] in absence of pre-stress (i.e. when $\Lambda_{i}=1$ ), has general solution given by

$$
F_{i d}^{\prime}=b_{i} t_{i} Y_{i}\left[\Lambda_{i} \cos \theta_{i d}^{\prime}-1+\sqrt{\left(1-\Lambda_{i} \cos \theta_{i d}^{\prime}\right)^{2}+\gamma_{i} \Lambda_{i}}\right],
$$

where $\gamma_{i}=2 R_{i} /\left(t_{i} Y_{i}\right)$ is a standard dimensionless parameter representing the ratio of adhesion resistance to tape extensional stiffness.

Within this general framework, the peeling process of a symmetrical multiple anchorage system, induced by vertical displacement, is described in what follows under the assumption of constitutively linear elements. Then, the special case of double peeling of two strips, forming a symmetrical Vshaped scheme, is studied in terms of critical pull-off event, by describing the quasi-static evolution of the tapes detachment, under the hypothesis of large deformations and nonlinear (specifically, neoHookean) hyperelasticity.

\section{Symmetrical peeling of linear multiple systems}

In the present section, a symmetrical scheme of multiple peeling is analyzed, in which $n / 2$ pairs of strips forming V-shaped arrangements, sharing the same geometrical and constitutive properties and storing the same level of pre-stretch in the adhering portions, undergo a prescribed pulling displacement in the vertical direction. In such a case, for any $i=1, \ldots, n / 2$, all the quantities related to the $i$-tape, can be assumed as coinciding with those characterizing the symmetrically positioned tape identified by the subscript $i+n / 2$; therefore, the subscript $i$, with $i=1, \ldots, n / 2$, will be used in this context to refer to the generic $i$-th pair of tapes constituting the anchorage system. Also, the geometrical relations in (2.1) as well as all the equations given in the previous section can be simplified by setting $\alpha=\pi / 2$.

For sake of simplicity and without loss of generality, the structure is here assumed as made by linearly elastic strips, whose strain energy function and nominal axial stress are those provided in equation (2.19). Hence, by following the Griffith criterion, symmetrical peeling of each pair of elements is ruled by equation (2.11) particularized for the case under exam as:

$$
\frac{\Psi_{i d}^{l i n}}{\Lambda_{i}}+P_{i d}^{l i n}\left(\frac{L_{i} \cos \Theta_{i}+d_{i}}{l_{i d}}-\frac{\lambda_{i d}}{\Lambda_{i}}\right)+\frac{R_{i}}{t_{i}}=0, \quad \forall i=1, \ldots, n / 2,
$$


where $\Psi_{i d}^{l i n}=\Psi_{i d}^{l i n}\left(u, d_{i}\right)=\left.\hat{\Psi}_{i}^{l i n}\right|_{\lambda_{i}=\lambda_{i d}}$ and $P_{i d}^{l i n}=P_{i d}^{l i n}\left(u, d_{i}\right)=\left.\hat{P}_{i}^{l i n}\right|_{\lambda_{i}=\lambda_{i d}}$, with $l_{i d}$ and $\lambda_{i d}$ written as

$$
\begin{aligned}
& l_{i d}=l_{i d}\left(u, d_{i}\right)=\sqrt{\left(d_{i}+L_{i} \cos \Theta_{i}\right)^{2}+\left(u+L_{i} \sin \Theta_{i}\right)^{2}} \\
& \lambda_{i d}=\lambda_{i d}\left(u, d_{i}\right)=\frac{\Lambda_{i} \sqrt{\left(d_{i}+L_{i} \cos \Theta_{i}\right)^{2}+\left(u+L_{i} \sin \Theta_{i}\right)^{2}}}{d_{i}+\Lambda_{i} L_{i}}, \quad \forall i=1, \ldots, n / 2
\end{aligned}
$$

according to the relations in (2.1). Since equation (3.1) does not provide analytical solution in terms of detached tape's portion as a function of the applied displacement, it is convenient, from the operational point of view, to rewrite it by assuming the axial stretch $\lambda_{i}$ within the tapes of the $i$-th pair as unknown, which results in

$$
\frac{\hat{\Psi}_{i}^{l i n}}{\Lambda_{i}}+\hat{P}_{i}^{l i n}\left(\frac{L_{i} \cos \Theta_{i}+d_{i}}{\lambda_{i} L_{i d}}-\frac{\lambda_{i}}{\Lambda_{i}}\right)+\frac{R_{i}}{t_{i}}=0 \quad \Leftrightarrow \quad \lambda_{i}^{3}+\sigma_{i} \lambda_{i}+\delta_{i}=0, \quad \forall i=1, \ldots, n / 2,
$$

the coefficients $\sigma_{i}$ and $\delta_{i}$ being defined as

$$
\sigma_{i}=-\frac{d_{i}\left(1+\gamma_{i} \Lambda_{i}+2 \Lambda_{i}^{2}\right)+L_{i} \Lambda_{i}\left(1+\gamma_{i} \Lambda_{i}+2 \Lambda_{i} \cos \Theta_{i}\right)}{d_{i}+L_{i} \Lambda_{i}}, \quad \delta_{i}=\frac{2 \Lambda_{i}^{2}\left(d_{i}+L_{i} \cos \Theta_{i}\right)}{d_{i}+L_{i} \Lambda_{i}} .
$$

Resolution of such equation thus gives the axial stretch $\tilde{\lambda}_{i d}$ arising within the $i$-th pair of tapes when detached for a tract $d_{i}$ as follows, if the parameters $\gamma_{i} \geq 0, \Lambda_{i} \geq 1$ and $\Theta_{i} \in[0, \pi / 2]$ are assigned:

$$
\tilde{\lambda}_{i d}=\tilde{\lambda}_{i d}\left(d_{i}\right)=\frac{\left(\sqrt{81 \delta_{i}^{2}+12 \sigma_{i}^{3}}-9 \delta_{i}\right)^{2 / 3}-12^{1 / 3} \sigma_{i}}{\left[18\left(\sqrt{81 \delta_{i}^{2}+12 \sigma_{i}^{3}}-9 \delta_{i}\right)\right]^{1 / 3}}, \quad \forall i=1, \ldots, n / 2 .
$$

Then, the corresponding value of applied displacement inducing detachment of the adhesive portion $d_{i}$ can be derived from equation $(3.2)_{2}$, that is:

$$
\tilde{u}_{i d}=\tilde{u}_{i d}\left(d_{i}\right)=\Lambda_{i}^{-1} \sqrt{\left(\tilde{\lambda}_{i d}^{l i n}\right)^{2}\left(d_{i}+L_{i} \Lambda_{i}\right)^{2}-\Lambda_{i}^{2}\left(d_{i}+L_{i} \cos \Theta_{i}\right)^{2}}-L_{i} \sin \Theta_{i}, \quad \forall i=1, \ldots, n / 2,
$$

whose critical threshold, at which peeling of the $i$-th pair of tapes begins and that satisfies the equation (2.12) in the present case, can be simply derived by setting $d_{i}=0$, i.e. $u_{i c r}=u_{i c r}\left(\Theta_{i}, \gamma_{i}, \Lambda_{i}, L_{i}\right)=$ $\left.\tilde{u}_{i d}\right|_{d_{i}=0}$. Then, the associated critical peeling angle can be obtained as

$$
\theta_{i c r}=\theta_{i c r}\left(\Theta_{i}, \gamma_{i}, \Lambda_{i}, L_{i}\right)=\left.\theta_{i e}\right|_{u=u_{i c r}}, \quad \theta_{i e}=\theta_{i e}(u)=\arctan \left[\left(L_{i} \sin \Theta_{i}+u\right) L_{i}^{-1} \sec \Theta_{i}\right],
$$

according to the equation (2.1).

On the other hand, by ideally considering endless tapes, when $d_{i} \rightarrow \infty$, the ratio $\tilde{u}_{i d} / d_{i}$ provides the finite value

$$
\begin{aligned}
\lim _{d_{i} \rightarrow \infty} \frac{\tilde{u}_{i d}}{d_{i}} & =\Lambda_{i}^{-1} \sqrt{\tilde{\lambda}_{i \infty}^{2}-\Lambda_{i}^{2}}, \\
\tilde{\lambda}_{i \infty} & =\frac{3^{1 / 3}\left(1+\gamma_{i} \Lambda_{i}+2 \Lambda_{i}^{2}\right)+\left[\sqrt{81 \Lambda_{i}^{4}-3\left(1+\gamma_{i} \Lambda_{i}+2 \Lambda_{i}^{2}\right)}-9 \Lambda_{i}^{2}\right]^{2 / 3}}{3^{2 / 3}\left[\sqrt{81 \Lambda_{i}^{4}-3\left(1+\gamma_{i} \Lambda_{i}+2 \Lambda_{i}^{2}\right)}-9 \Lambda_{i}^{2}\right]^{1 / 3}},
\end{aligned}
$$

this ensuring that, in the limit in which one applies an infinite displacement to the system's hinge, namely a displacement much higher then the length of the longest tape, the detached portions of all the elements increase proportionally to the magnitude of the same displacement with a rate that 
depends on the adhesion parameter $\gamma_{i}$ and the pre-stretch stored in the adherent portions $\Lambda_{i}$, while it is not influenced by the initial orientation $\Theta_{i}$. In this regard, it is worth underlying that the asymptotic peeling configuration of the system turns out to lose any track about the angles described by the tapes in their reference arrangement, as also observable for the quantities derived below. In particular, one can find that, for this symmetrical and constitutively linear model, the axial force borne by the $i$-th pair of tapes in the limit of $u \rightarrow \infty\left(d_{i} \rightarrow \infty\right)$ is given, according to its general expression (2.14), by

$$
\tilde{F}_{i \infty}=\lim _{u \rightarrow \infty} \tilde{F}_{i}=b_{i} t_{i} Y_{i}\left(\tilde{\lambda}_{i \infty}-1\right)
$$

while, due to equations (2.15) and (2.1), the corresponding actual limit inclination can be calculated as

$$
\tilde{\theta}_{i \infty}=\lim _{u \rightarrow \infty} \tilde{\theta}_{i}=\arctan \left[\Lambda_{i}^{-1} \sqrt{\tilde{\lambda}_{i \infty}^{2}-\Lambda_{i}^{2}}\right] .
$$

As a consequence, the limit value of the total pull-off force, lying in the present case in the vertical direction, is provided by exploiting equation (2.13) as:

$$
\tilde{F}_{\infty}=\lim _{u \rightarrow \infty} \tilde{F}=2 \sum_{i=1}^{n / 2} \tilde{F}_{i \infty} \sin \tilde{\theta}_{i \infty}=2 \sum_{i=1}^{n / 2} b_{i} t_{i} Y_{i} \frac{\left(\tilde{\lambda}_{i \infty}-1\right) \sqrt{\tilde{\lambda}_{i \infty}^{2}-\Lambda_{i}^{2}}}{\tilde{\lambda}_{i \infty}} .
$$

By now dealing with an actual case, let assume

$$
\Lambda_{i}=1, R_{i}=R, t_{i}=t, Y_{i}=Y, \quad \forall i=1, \ldots, n / 2,
$$

this also guaranteeing

$$
\gamma_{i}=\gamma=2 R /(t Y), \quad \forall i=1, \ldots, n / 2 .
$$

Additionally, geometrical compatibility allows to write:

$$
L_{i}=\frac{\sin \Theta_{1}}{\sin \Theta_{i}} L_{1}, \quad \forall i=2, \ldots, n / 2,
$$

while the imposition that the initially free (i.e. not adhering) portions of the strips have equal reference volume, say $V_{i e}=V_{1 e}$, leads to the relation:

$$
b_{i}=\frac{\sin \Theta_{i}}{\sin \Theta_{1}} b_{1}, \quad \forall i=2, \ldots, n / 2 .
$$

Under these assumptions, by evaluating the critical solution $u_{i c r}$ as described above, one obtains that, for any value of $\gamma$, it results a decreasing function of the angle $\Theta_{i}$ ranging within the compatible interval $[0, \pi / 2]$. This implies that, since the index $i$ grows from the left to the right of the considered anchorage scheme (see figure $2 \mathrm{~A}$ by way of example) and thus $\Theta_{i} \leq \Theta_{i+1} \forall i=2, \ldots, n / 2$, the relationship $u_{i c r} \geq u_{(i+1) c r}$ holds true $\forall i=2, \ldots, n / 2$, so that the innermost pair of strips is always the first one to undergo delamination, namely $u_{c r}=u_{(n / 2) c r}=\min _{i} u_{i c r}$.

On this basis, figure $3 \mathrm{~A}$ shows the evolution of the delamination tracts $\tilde{d}_{i}$ in function of an increasing prescribed displacement $u$, as obtained by numerically inverting the relation (3.6) particularized for the present case of study, when assuming, by way of example, that $n=6$ and that the initial inclinations of the tapes are $\Theta_{1}=\pi / 6, \Theta_{2}=\pi / 4$ and $\Theta_{3}=\pi / 3$. After a purely elastic first phase $\left(\tilde{d}_{i}=0 \forall i=1,2,3\right)$, it is possible to observe the progressive insurgence the tapes detachment (at points referred to as 2,3 and 4), from the most to the less inclined pair (i.e. from $i=3$ to $i=1$ ), when the applied displacement reaches the corresponding critical values $u_{i c r}$. Also, one can notice how the curves tend to become parallel straight lines when sufficiently increasing $u$, in compliance with the result shown in equation (3.8) where the dependence on the index $i$ disappears for the present 

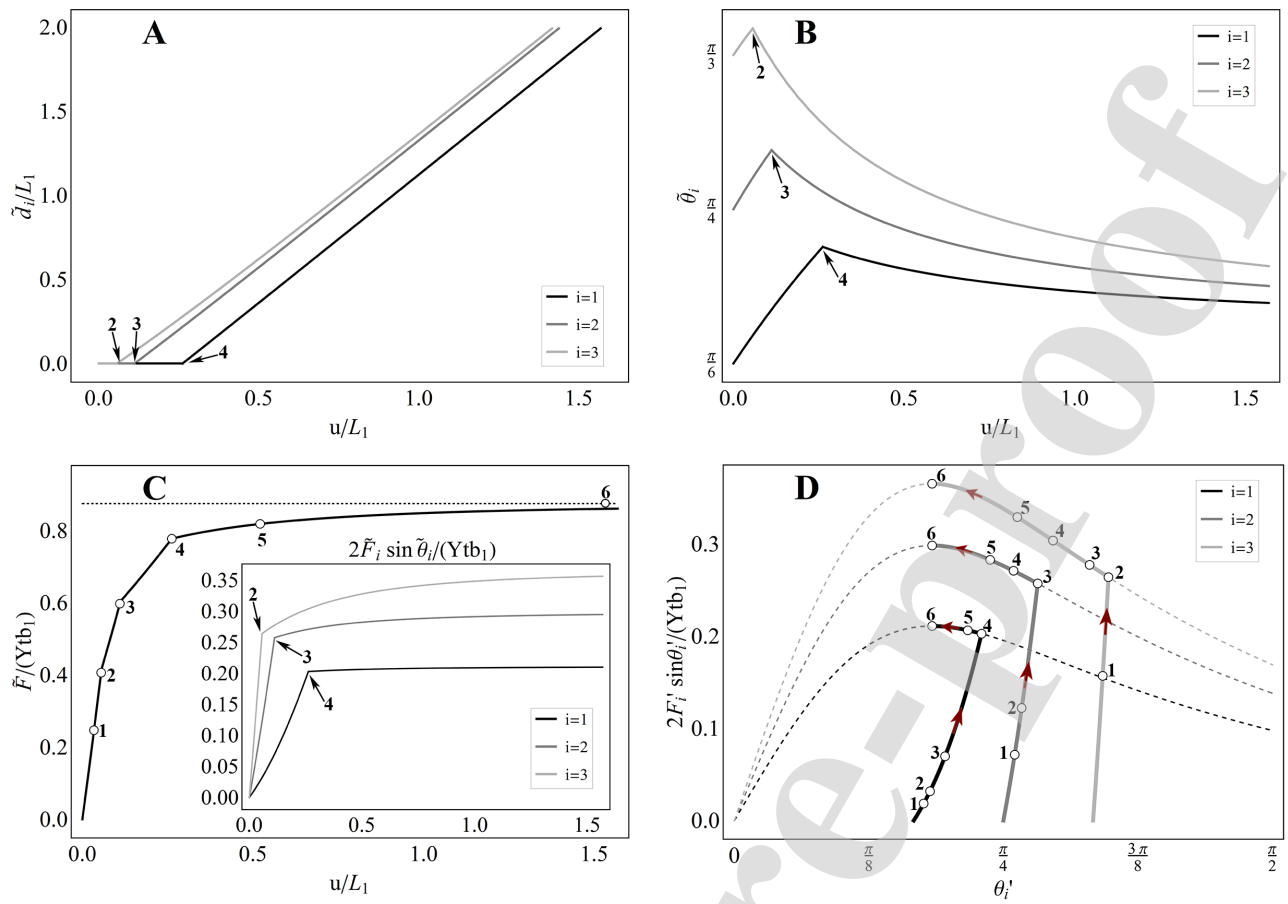

Figure 3: A) Evolution of the normalized lengths of the detached portions of the tapes $\left(\tilde{d}_{i} / L_{1}\right)$ when increasing the normalized magnitude of the vertical pulling displacement $\left(u / L_{1}\right)$ applied at the free hinge of the symmetrical scheme of multiple peeling analyzed in the present section, with $n=6$ and $\Theta_{1}=\pi / 6, \Theta_{2}=\pi / 4$, $\Theta_{3}=\pi / 3$. B) Trend of the current angles $\tilde{\theta}_{i}$ described by the three pairs of tapes with respect to the horizontal direction while pulling the system. C) Normalized total pull-off force $\tilde{F} /\left(Y t b_{1}\right)$ corresponding to growing externally prescribed displacement, with -in the inset- the single contributions given to it by each pair of tapes. D) Trend of the normalized current contributions $2 F_{i}^{\prime} \sin \theta_{i}^{\prime} /\left(Y t b_{1}\right)$ given to the vertical peeling force by the single pairs of strips as function of their current inclinations $\theta_{i}^{\prime}$. Herein, the dashed curves are those representing the solution of the problem written with respect to the current configuration, hence those derived from the solution of the Kendall's equation. On the other hand, the thick solid lines identify the curves portions traced (in the red arrows direction) by the pairs of values of forces and angles actually experienced by the tapes while increasing the pulling displacement prescribed with respect to the reference configuration of the system. In all these plots, numbers from 1 to 6 indicate the next representative phases of the peeling process: at point 1 all the tapes deform elastically without detaching; at point 2 the innermost tape $(i=3)$ first begins to detach while the others still respond as purely elastic; at points 3 and 4 the second $(i=2)$ and the third $(i=1)$ pairs of elements also undergo delamination, respectively, so that at point 5 all the strips have a detached portion; finally, point 6 identify the limit asymptotic configuration corresponding to an infinite applied displacement, that is elements all detached for an infinite portion.

application, in which $\gamma_{i}=\gamma$ and $\Lambda_{i}=1$. The contemporary evolution of the current orientations of the tapes $\tilde{\theta}_{i}$ in response to growing pulling displacement, evaluated according to the general equation (2.15), is then illustrated in figure $3 \mathrm{~B}$. Specifically, starting from the assigned value $\Theta_{i}$, each angle $\tilde{\theta}_{i}$ grows during the strip's elastic response up to a maximum corresponding to the critical peeling angle $\theta_{i c r}$ given in (4.6) and then decreases by asymptotically approaching the limit value $\theta_{i \infty}$ provided in (3.10), in this case coinciding for all tapes, say $\theta_{i \infty}=\theta_{\infty} \forall i=1,2,3$.

On the other hand, it is worth highlighting the softening behaviour that the overall system exhibits while pulling it off as a consequence of the progressive detachment of its elements, as presented in 
figure 3C, where both the total pull-off force $\tilde{F}$ and the vertical contributions given to it by the single pairs of tapes, computed on the basis of equations (2.13)-(2.15), are plotted. Therein, the asymptotic limits to which these quantities tend for growing $u$ are those deriving from equations (3.9)-(3.11).

Finally, the thick solid lines in figure 3D show the response of the system's single pairs of elements in terms of their current contributions to the total pull-off force (say $2 F_{i}^{\prime} \sin \theta_{i}^{\prime}$ ) versus the associated current inclination $\left(\theta_{i}^{\prime}\right)$. In compliance with results of figures $3 \mathrm{~B}-3 \mathrm{C}$, the path followed in this plane by each tape under a growing applied displacement is the one indicated by the direction of the arrows, characterized by increasing inclination and vertical force during the purely elastic deformation phase up to the respective critical values inducing first detachment, while by decreasing inclination up to a lower limit and increasing force up to an upper bound during the peeling progression. In particular, the curves described during the purely elastic response of the tapes can be analytically expressed by replacing $F_{i}^{\prime}$ with

$$
F_{i e}^{\prime}=F_{i e}^{\prime}\left(\theta_{i}^{\prime}\right)=b_{i} t_{i} Y_{i}\left(\lambda_{i e}^{\prime}-1\right)=b_{1} t Y \frac{\sin \Theta_{i}}{\sin \Theta_{1}}\left(\frac{\cos \Theta_{i}}{\cos \theta_{i}^{\prime}}-1\right), \quad \text { where } \quad \lambda_{i e}^{\prime}=\lambda_{i e}^{\prime}\left(\theta_{i}^{\prime}\right)=\frac{\cos \Theta_{i}}{\cos \theta_{i}^{\prime}},
$$

is the corresponding longitudinal stretch borne by each strip in the undetached configuration. On the other hand, the curves traced in the post-critical phase de facto overlie those related to the solution of the generalized Kendall equation as given in (2.22) for $\Lambda_{i}=1$ (see dashed tracts in figure 3D), which indeed in general describes the linear peeling problem at the critical event and during the quasi-static evolution by referring it to the current configuration in terms of axial force versus current peeling angle. Therefore, in this phase, the expression of $F_{i}^{\prime}$ coincides with

$$
\tilde{F}_{i d}^{\prime}=b_{1} t Y \frac{\sin \Theta_{i}}{\sin \Theta_{1}}\left[\cos \theta_{i}^{\prime}-1+\sqrt{\left(1-\cos \theta_{i}^{\prime}\right)^{2}+\gamma}\right] \text {. }
$$

Then, the imposition of the equality $F_{i e}^{\prime}=\tilde{F}_{i d}^{\prime}$, provides the critical peeling angle of each strip, that is the intersection of the two curves, as a function of the adhesion parameter $\gamma$ and of the reference slope $\Theta_{i}$, which coincides with the critical peeling angle $\theta_{i c r}$ derived for the present application from the solution in equation (4.6), obtained by writing the problem with respect to the initial configuration of the system as described above. On the other hand, calculation of the point of maximum of the function $2 \tilde{F}_{i d}^{\prime} \sin \theta_{i}^{\prime}$ with respect to the current angle $\theta_{i}^{\prime}$ provides the value $\theta_{i \infty}$ already obtained above as asymptotic limit to which the peeling angle of the $i$-th pair of ideally endless tapes tends when $u \rightarrow \infty\left(d_{i} \rightarrow \infty\right)$.

\section{Double peeling of nonlinear V-shaped systems}

In the present section, the double peeling process of the V-shaped symmetrical system illustrated in figure $2 \mathrm{~B}$ is analyzed. By starting from the above studied multiple anchorage scheme, a pair of two strips, having the same geometrical and constitutive properties and storing the same level of prestretch in the adhering portions, are peeled from the rigid ground by applying a vertical displacement, therefore the geometrical relations provided in equation (2.1) can be in this case further simplified both by assuming $\alpha=\pi / 2$ and by removing the subscript $i$.

\subsection{Critical peeling event}

By following the Griffith criterion [28], symmetrical peeling of the two strip elements initiates at a critical value of applied displacement, $u_{c r}$, that satisfies the equation reported below, particularized 
for the specific case from the general condition in (2.12):

$$
-\left.\frac{\partial \Pi_{d}}{\partial d}\right|_{d=0}=b R \quad \Leftrightarrow \quad \frac{\Psi_{e}}{\Lambda}+P_{e}\left(\frac{\cos \Theta}{\lambda_{e}}-\frac{\lambda_{e}}{\Lambda}\right)+\frac{R}{t}=0,
$$

where $\Psi_{e}=\Psi_{e}(u)$ and $P_{e}=P_{e}(u)$ are functions of $u$ according to their definitions given in section 2, and

$$
\lambda_{e}=\lambda_{e}(u)=L^{-1} \sqrt{L^{2}+2 L u \sin \Theta+u^{2}} .
$$

For sake of simplicity, equation $(4.1)_{2}$ can be rewritten by assuming the axial stretch $\lambda$ of the two tapes as unknown, i.e.

$$
\frac{\hat{\Psi}}{\Lambda}+\hat{P}\left(\frac{\cos \Theta}{\lambda}-\frac{\lambda}{\Lambda}\right)+\frac{R}{t}=0
$$

with $\hat{\Psi}=\hat{\Psi}(\lambda)$ and $\hat{P}=\hat{P}(\lambda)$, in a way that solution of such equation with respect to $\lambda$ provides the value of the critical stretch $\lambda_{c r}$ occurring within the elements at their first detachment as a function of their constitutive and adhesive properties, of the stored pre-stretch and of the system's geometry in the reference configuration. Then, the corresponding critical displacement can be derived from equation (4.2) as:

$$
u_{c r}=\left(\sqrt{\lambda_{c r}^{2}+\sin ^{2} \Theta-1}-\sin \Theta\right) L,
$$

while the adhesion strength of the system, in compliance with its general expression given in (2.16), is provided by

$$
F_{c r}=2 b t P_{c r} \sin \theta_{c r},
$$

where $P_{c r}$ is the critical nominal axial stress for each tape, i.e. $P_{c r}=\left.P_{e}\right|_{u=u_{c r}}=\left.\hat{P}\right|_{\lambda=\lambda_{c r}}$, and $\theta_{c r}$ is the critical peeling angle following from equation (2.1) as

$$
\theta_{c r}=\left.\theta_{e}\right|_{u=u_{c r}}, \quad \theta_{e}=\theta_{e}(u)=\arctan \left[(L \sin \Theta+u) L^{-1} \sec \Theta\right] .
$$

Specifically, by making the hypothesis that the system is made of hyperelastic strips obeying the neo-Hookean law and behaving as incompressible elements (having for example in mind rubber-like materials), the related free energy density and longitudinal first Piola-Kirchhoff stress can be written as [51]:

$$
\hat{\Psi}_{\text {neo }}=\hat{\Psi}_{\text {neo }}(\lambda)=\frac{Y}{6} \frac{\lambda^{3}-3 \lambda+2}{\lambda}, \quad \hat{P}_{\text {neo }}=\hat{P}_{\text {neo }}(\lambda)=\frac{Y}{3}\left(\lambda-\lambda^{-2}\right) .
$$

By hence replacing these expressions into equation (4.3), it finally leads to:

$$
\lambda^{5}+(3-3 \gamma \Lambda-2 \Lambda \cos \Theta) \lambda^{3}-4 \lambda^{2}+2 \Lambda \cos \Theta=0 .
$$

As well known, according to the Abel-Ruffini theorem, no algebraic solutions exist for general quintic (or higher degree) polynomial equations with arbitrary coefficients. This is actually the case of equation (4.8), therefore its solution, say $\tilde{\lambda}_{c r}=\tilde{\lambda}_{c r}(\Theta, \gamma, \Lambda)$, has been found as a function of assigned values of the free parameters of the system via numerical methods ${ }^{1}$, with the aid of the symbolic code Mathematica [52]. Then, the corresponding critical quantities $\tilde{u}_{c r}, \tilde{F}_{c r}$ and $\tilde{\theta}_{c r}$ have been in cascade obtained for this case of neo-Hookean strips by properly resorting to expressions given in (4.4), (4.5)

\footnotetext{
${ }^{1}$ It is worth to note that algebraic solutions could be derived for equation (4.8) if the parameters $\Theta, \Lambda$ and $\gamma$ were related in a way to make it a reciprocal equation of first type (the second type would not be admissible in the present case), namely in the special case such that its coefficients were related symmetrically as:

$$
2 \Lambda \cos \Theta=1 \text { and } 3-3 \gamma \Lambda-2 \Lambda \cos \Theta=-4 \quad \Leftrightarrow \quad \cos \Theta=\frac{1}{2 \Lambda} \text { and } \gamma=\frac{2}{\Lambda}, \quad \forall \Lambda \geq 1 .
$$
}


and (4.6), respectively. In particular, closed-form solution can be obtained, for any pair $(\gamma, \Lambda)$, only in the limit case of initially vertical tapes, that is $\Theta \rightarrow \pi / 2$, for which the constant term of equation (4.8) vanishes, thus allowing to analytically derive:

$$
\tilde{\lambda}_{c r}^{\pi / 2}=\tilde{\lambda}_{c r}^{\pi / 2}(\gamma, \Lambda)=\left.\tilde{\lambda}_{c r}\right|_{\Theta \rightarrow \pi / 2}=x+\frac{\gamma \Lambda-1}{x}, \quad x=(2+\sqrt{5-\gamma \Lambda[3+\gamma \Lambda(\gamma \Lambda-3)]})^{1 / 3}
$$

and the related solutions $\tilde{u}_{c r}^{\pi / 2}, \tilde{F}_{c r}^{\pi / 2}$ and $\tilde{\theta}_{c r}^{\pi / 2}$ via equations (4.4), (4.5) and (4.6), respectively. On the other hand, approximated analytical solution of equation (4.8) can be de facto gained from the knowledge of the analytical solution of the problem under the assumption of linear tapes, which derives as special case of the symmetrical multiple peeling model described in the previous section, that is:

$$
\begin{aligned}
& \lambda_{c r}^{l i n}=\lambda_{c r}^{l i n}(\Theta, \gamma, \Lambda)=3^{-2 / 3}\left(\varphi+\frac{\xi}{\varphi}\right) \\
& \xi=3^{1 / 3}(1+\gamma \Lambda+2 \Lambda \cos \Theta), \varphi=\left[\sqrt{(9 \Lambda \cos \Theta)^{2}-\xi^{3}}-9 \Lambda \cos \Theta\right]^{1 / 3},
\end{aligned}
$$

the associated critical displacement $u_{c r}^{l i n}$, adhesion strength $F_{c r}^{l i n}$ and critical peeling angle $\theta_{c r}^{l i n}$ being given by equations (4.4), (4.5) and (4.6), respectively. Then, a good approximation of the critical elastic stretch for the neo-Hookean strips, say $\tilde{\lambda}_{c r}^{a p x}$, can be found by solving the third-order Taylor's series expansion of the Griffith equation (4.8) with respect to $\lambda_{c r}^{l i n}$, which provides:

$$
\begin{aligned}
\tilde{\lambda}_{c r}^{a p x}= & \tilde{\lambda}_{c r}^{a p x}(\Theta, \gamma, \Lambda)=\frac{1}{3}\left\{\frac{2^{1 / 3}\left(\omega^{2}-3 \eta\right)}{\left[9 \omega \eta-2 \omega^{3}-27 \kappa+3 \sqrt{3 \eta^{2}\left(4 \eta-\omega^{2}\right)+6 \omega \kappa\left(2 \omega^{2}-9 \eta\right)+81 \kappa^{2}}\right]^{1 / 3}},\right. \\
& \left.+2^{-1 / 3}\left[9 \omega \eta-2 \omega^{3}-27 \kappa+3 \sqrt{3 \eta^{2}\left(4 \eta-\omega^{2}\right)+6 \omega \kappa\left(2 \omega^{2}-9 \eta\right)+81 \kappa^{2}}\right]^{1 / 3}-\omega\right\}
\end{aligned}
$$

where the coefficients $\omega, \eta$ and $\kappa$ are written as follows:

$$
\begin{gathered}
\omega=-\frac{4\left[1+5\left(\lambda_{c r}^{l i n}\right)^{3}\right]}{3-3 \gamma \Lambda+10\left(\lambda_{c r}^{l i n}\right)^{2}-2 \Lambda \cos \Theta}, \\
\eta=\frac{15\left(\lambda_{c r}^{l i n}\right)^{4}}{3-3 \gamma \Lambda+10\left(\lambda_{c r}^{l i n}\right)^{2}-2 \Lambda \cos \Theta}, \\
\kappa=\frac{2 \Lambda \cos \Theta-4\left(\lambda_{c r}^{l i n}\right)^{5}}{3-3 \gamma \Lambda+10\left(\lambda_{c r}^{l i n}\right)^{2}-2 \Lambda \cos \Theta} .
\end{gathered}
$$

It is possible to verify that an error less than $0.5 \%$ exists between the numerical solution of the equation (4.8) and the approximated one for values of the system's parameters ranging within realistic intervals, say $\Theta \in[0, \pi / 2], \Lambda \in[1,2]$ and $\gamma \in[0,1]$, the latter being compatible with the orders of magnitude usually measured in literature experiments. On these bases, analytical expressions can be also obtained for the critical displacement $\tilde{u}_{c r}^{a p x}$, the critical pull-off force $\tilde{F}_{c r}^{a p x}$ and the critical peeling angle $\tilde{\theta}_{c r}^{a p x}$ by simply substituting $\tilde{\lambda}_{c r}^{a p x}$ in equations (4.4), (4.5) and (4.6).

In particular, analysis of the first and second derivatives of the adhesion strength $\tilde{F}_{c r}^{a p x}$ with respect to the initial inclination of the tapes $\Theta$ shows that optimal (namely, maximum) adhesion occurs in the case of a flat system, i.e. when $\Theta=0, \tilde{F}_{c r}^{0}=\left.\tilde{F}_{c r}\right|_{\Theta=0}\left(\tilde{F}_{0}^{a p x}=\left.\tilde{F}_{c r}^{a p x}\right|_{\Theta=0}\right)$ in fact representing the highest critical pull-off force over the whole range of $\Theta \in[0, \pi / 2]$, for any chosen set of the other model's parameters, as observable, by way of example, in figure 4A, obtained for fixed $\Lambda=1.1$ and 

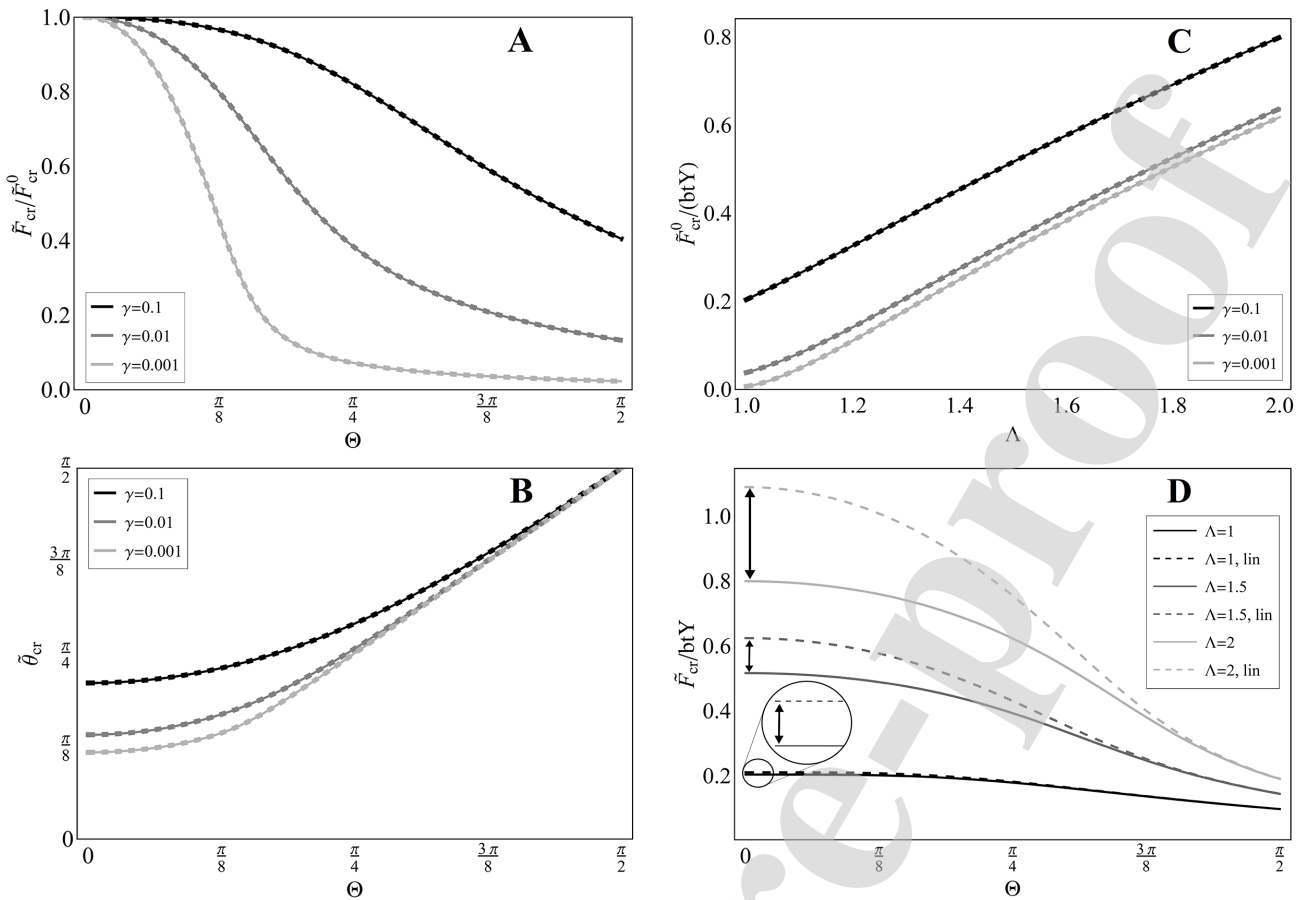

Figure 4: A) Critical pull-off force $\tilde{F}_{c r}$-normalized to its (maximum) value $\tilde{F}_{c r}^{0}$ - and B) critical peeling angle $\tilde{\theta}_{c r}$, both plotted versus the reference tapes' inclination angle $\Theta$, for different values of the parameter $\gamma$ and fixed pre-stretch $\Lambda=1.1$. C) Normalized optimal critical pull-off force $\tilde{F}_{c r}^{0} /(b t Y)$ versus pre-stretch $\Lambda$ for different values of the adhesion parameter $\gamma$. In these three plots, dashed curves illustrate the numerically obtained solutions while the solid tracts correspond to the analytical approximated ones, say $\tilde{F}_{c r}^{a p x}, \tilde{\theta}_{c r}^{a p x}$ and $\tilde{F}_{0}^{a p x}$. D) Solid curves show the normalized critical pull-off force $\tilde{F}_{c r} /(b t Y)$ versus the reference inclination angle $\Theta$, obtained for $\gamma=0.1$ and different values of $\Lambda$, compared with the one exhibited in case of linear tapes (dashed lines).

varying $\gamma$, and in the solid curves of figure 4D, obtained for prescribed $\gamma=0.1$ and different values of $\Lambda$. It is worth noting that such a result reproduces that also obtained for the case of linear constitutive assumption, as evidenced in the dashed curves of figure 4D and in previous literature papers [35]. On the other hand, it is found that, for any assigned reference inclination $\Theta$, both the critical peeling force $\tilde{F}_{c r}\left(\tilde{F}_{c r}^{a p x}\right)$ and the related critical angle $\tilde{\theta}_{c r}\left(\tilde{\theta}_{c r}^{a p x}\right)$ are increasing functions of $\gamma$ and $\Lambda$ (see figure 4 ), both the adhesion parameter and the initially stored pre-stretch hence contributing to delay the first detachment event.

To highlight the importance of employing nonlinear hyperelastic models when highly deformable materials are considered and large strain regimes can be already reached before detachment is triggered, in figure 4D, the values of the (normalized) critical pull-off load obtained in the present work by assuming neo-Hookean strips are compared with the ones resulting from linear constitutive assumptions. A certain discrepancy is thus revealed between the two models, which grows by increasing the pre-stretch $\Lambda$ and narrows while varying $\Theta$ from 0 (condition of maximum adhesion) to $\pi / 2$, for any selected $\gamma$. According to these outcomes, the linear assumption leads to overestimate the critical force with respect to the neo-Hookean model, this effect being likely related to the softening that characterizes the one-dimensional nominal stress-stretch curve of (incompressible) neo-Hookean materials at finite elongations with respect to the initial (intrinsic) stiffness. The order relation could be therefore possibly inverted and, in general, quantitatively different, by adopting other constitutive laws, for ex- 
ample characterized by stiffness hardening for growing deformation levels.
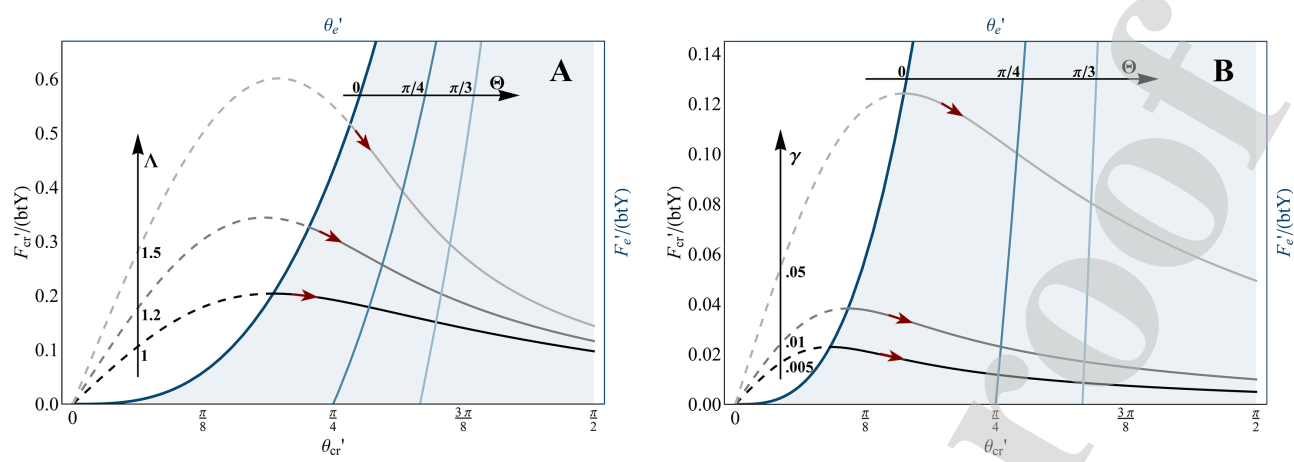

Figure 5: In the blue-coloured curves, the trend of the normalized pull-off force $F_{e}^{\prime} /(b t Y)$ versus the tapes inclination $\theta_{e}^{\prime}$ corresponding to the purely elastic response of the V-shaped system, for different initial inclinations $\Theta=0, \pi / 4, \pi / 3$. In the grey-coloured curves, the trend of the normalized critical pull-off force $F_{c r}^{\prime} /(b t Y)$ versus the critical peeling angle $\left.\theta_{c r}^{\prime}, \mathbf{A}\right)$ for a fixed value of $\gamma=0.1$ and different levels of stored pre-stretch $\Lambda=1,1.2,1.5$ and $\mathbf{B}$ ) for fixed $\Lambda=1$ and varying $\gamma=0.005,0.01,0.05$. The solid tracts of the gray-coloured lines contain the pair of critical forces and angles that can be actually experienced by the system when increasing its reference inclination $\Theta$ from 0 to $\pi / 2$ (in the direction of the red arrows), while the dashed portions correspond to incompatible critical configurations.

Finally, the response of the system is shown in figure 5A in terms of pull-off force as a function of the inclination of the tapes, both at the critical event and during the purely elastic phase. Specifically, the grey-coloured solid curves describe the relation between the critical pull-off force and the corresponding critical peeling angle as obtained above, for a fixed value of $\gamma$ and different levels of stored pre-stretch $\Lambda$, while considering $\Theta$ as growing from 0 to $\pi / 2$ (in the direction of the red arrows). However, such relation can be directly obtained, as anticipated in section 2, by alternatively setting the problem with respect to the current configuration of the system by following equation (2.18), which in the present case gives:

$$
\frac{\hat{\Psi}_{n e o}}{\Lambda}+\hat{P}_{n e o}\left(\cos \theta-\frac{\lambda}{\Lambda}\right)+\frac{R}{t}=0 \Leftrightarrow \lambda^{4}-2 \Lambda \cos \theta\left(\lambda^{3}-1\right)+3(1-\gamma \Lambda) \lambda^{2}-4 \lambda=0,
$$

herein $\theta$ and $\lambda$ respectively referring to the actual inclination and stretch of the strips in the current configuration. Analytical solution of such equation then provides the relation between the axial stretch $\lambda_{c r}^{\prime}$ and the inclination $\theta_{c r}^{\prime}$ of the tapes at the first detachment event for assigned values of $\Lambda$ and $\gamma$, neglecting any information about the initial configuration of the system, that is independently from $\Theta$. Specifically, such relation can be expressed as follows

$$
\begin{aligned}
\lambda_{c r}^{\prime}= & \lambda_{c r}^{\prime}\left(\theta_{c r}^{\prime}\right)=\frac{\Lambda \cos \theta_{c r}^{\prime}}{2}+\tau+ \\
& \frac{1}{2} \sqrt{\frac{4-3 \Lambda(1-\gamma \Lambda) \cos \theta_{c r}^{\prime}+\Lambda^{3}\left(\cos \theta_{c r}^{\prime}\right)^{3}}{\tau}-4 \tau^{2}-6\left[1-\gamma \Lambda-\frac{\Lambda^{2}\left(\cos \theta_{c r}^{\prime}\right)^{2}}{2}\right]},
\end{aligned}
$$


where

$$
\begin{aligned}
& \tau=\frac{1}{2} \sqrt{\Lambda^{2}\left(\cos \theta_{c r}^{\prime}\right)^{2}-2(1-\gamma \Lambda)+\frac{\Delta}{3}+\frac{3(1-\gamma \Lambda)^{2}}{\Delta}}, \\
& \Delta=\left[\frac{\varphi+\sqrt{\varphi^{2}-4(3-3 \gamma \Lambda)^{6}}}{2}\right]^{1 / 3}, \\
& \varphi=432+54(1-\gamma \Lambda)^{3}-648 \Lambda(3-3 \gamma \Lambda) \cos \theta_{c r}^{\prime}+216 \Lambda^{3}\left(\cos \theta_{c r}^{\prime}\right)^{3}
\end{aligned}
$$

As a consequence, the relation between the system's adhesion strength and the critical peeling angle is:

$$
F_{c r}^{\prime}=F_{c r}^{\prime}\left(\theta_{c r}^{\prime}\right)=\frac{2}{3} b t Y\left[\lambda_{c r}^{\prime}-\left(\lambda_{c r}^{\prime}\right)^{-2}\right] \sin \theta_{c r}^{\prime},
$$

which provides (for the considered values of $\gamma$ and $\Lambda$ ) the grey-coloured curves in figure 5A over the whole range $\theta_{c r}^{\prime} \in[0, \pi / 2]$, whose only compatible tracts, actually referring to initial inclinations $\Theta$ of the tapes comprised within the range $[0, \pi / 2]$, are the solid ones, as described above. Then, since the function in equation (4.16) links critical peeling forces and angles neglecting which is the actual reference configuration of the system leading to them, for tracing it back, one has to impose the intersection of the curve $F_{c r}^{\prime}\left(\theta_{c r}^{\prime}\right)$ with the one describing the evolution of the pull-off force in the purely elastic phase, say $F_{e}^{\prime}$, as a function of the current inclination of the tapes $\theta_{e}^{\prime}$, that is

$$
F_{e}^{\prime}=F_{e}^{\prime}\left(\theta_{e}^{\prime}\right)=\frac{2}{3} b t Y\left[\lambda_{e}^{\prime}-\left(\lambda_{e}^{\prime}\right)^{-2}\right] \sin \theta_{e}^{\prime}, \quad \text { where } \quad \lambda_{e}^{\prime}=\lambda_{e}^{\prime}\left(\theta_{e}^{\prime}\right)=\frac{\cos \Theta}{\cos \theta_{e}^{\prime}},
$$

is the corresponding longitudinal stretch borne by each strip, whose expression in terms of the reference and current inclinations derive from the geometrical relationship $L \cos \Theta=l_{e}^{\prime} \cos \theta_{e}^{\prime}, l_{e}^{\prime}$ being the current length of the tapes in the undetached configuration. The relation in equation (4.17) is graphically illustrated in the blue-coloured curves of figure 5A for different values of $\Theta$. Then, the imposition of the equality of the forces $F_{e}^{\prime}$ and $F_{c r}^{\prime}$ allows to numerically determine the critical peeling angle and, as a result, the critical load as functions of the initial inclination of the tapes $\Theta$, which respectively correspond to the values $\tilde{\theta}_{c r}$ and $\tilde{F}_{c r}$ derived above by writing the problem with respect to the initial configuration of the anchorage system, and that are hence approximated by the analytical expressions $\tilde{\theta}_{c r}^{a p x}$ and $\tilde{F}_{c r}^{a p x}$. In particular, in compliance with the results previously highlighted, the curve $\left.F_{e}^{\prime}\left(\theta_{e}^{\prime}\right)\right|_{\Theta=0}$ is the one containing the critical pull-off load maxima $\tilde{F}_{c r}^{0}$, which can be therefore equivalently derived by requiring $F_{c r}^{\prime}=\left.F_{e}^{\prime}\right|_{\Theta=0}$. Additionally, it is possible to find that in case of absence of pre-stress in the adherent portions of the tapes (i.e. $\Lambda=1$ ), the adhesion strength maxima obtained for varying $\gamma$ coincide with the stationary points of maximum of the curves $\left.F_{c r}^{\prime}\left(\theta_{c r}^{\prime}\right)\right|_{\Lambda=1}$ corresponding to the same values of the adhesion parameter, which in fact all lie over the curve $\left.F_{e}^{\prime}\right|_{\{\Theta=0, \Lambda=1\}}$, as shown in figure 5B. On the other hand, this does not hold true when the system stores a value of pre-stretch $\Lambda>1$.

\subsection{Detachment progression}

The quasi-static evolution of the detachment process of the hyperelastic V-shaped adhesive system, developing after the critical peeling event, is analyzed in what follows. To this aim, the law describing the progress of the delamination tract $d$ is determined for growing prescribed displacement beyond the critical threshold.

Specifically, by applying the Griffith criterion for the present case of symmetrical double peeling, the 
problem can be formulated as:

$$
-\frac{\partial \Pi_{d}}{\partial d}=b R \Leftrightarrow \frac{\hat{\Psi}_{n e o}\left(\lambda_{d}\right)}{\Lambda}+\hat{P}_{n e o}\left(\lambda_{d}\right)\left[\frac{\Lambda(L \cos \Theta+d)}{\lambda_{d}(\Lambda L+d)}-\frac{\lambda_{d}}{\Lambda}\right]+\frac{R}{t}=0,
$$

$\lambda_{d}=\lambda_{d}(u, d)$ coinciding with that given in (3.2) 2 when removing the subscript $i$. Since closed-form solution of such equation cannot be earned, the delamination function $\tilde{d}=\tilde{d}(u)$ has been found numerically by the aid of Mathematica [52], $\forall u \geq \tilde{u}_{c r}$. On these bases, the resulting vertical peeling force has been determined according to the following expression:

$$
\tilde{F}_{d}=\tilde{F}_{d}(u)=2 b t \hat{P}_{n e o}\left(\tilde{\lambda}_{d}\right) \sin \tilde{\theta}_{d}, \quad \forall u \geq \tilde{u}_{c r},
$$

where $\tilde{\lambda}_{d}=\tilde{\lambda}_{d}(u)=\left.\lambda_{d}\right|_{d=\tilde{d}}$ and the peeling angle $\tilde{\theta}_{d}$ is given by

$$
\tilde{\theta}_{d}=\tilde{\theta}_{d}(u)=\arctan \left[\frac{L \sin \Theta+u}{L \cos \Theta+\tilde{d}}\right], \quad \forall u \geq \tilde{u}_{c r} .
$$

Therefore, the pull-off force $\tilde{F}$, offered by the anchorage system during the whole process, from the purely elastic phase to the one involving delamination, has been then obtained as

$$
\tilde{F}=\tilde{F}(u)=F_{e}\left(H\left(\tilde{u}_{c r}-u\right)-\frac{1}{2} \delta\left(\tilde{u}_{c r}-u\right)\right)+\tilde{F}_{d}\left(H\left(u-\tilde{u}_{c r}\right)-\frac{1}{2} \delta\left(\tilde{u}_{c r}-u\right)\right)
$$

and the related inclination angle as

$$
\tilde{\theta}=\tilde{\theta}(u)=\theta_{e}\left(H\left(\tilde{u}_{c r}-u\right)-\frac{1}{2} \delta\left(\tilde{u}_{c r}-u\right)\right)+\tilde{\theta}_{d}\left(H\left(u-\tilde{u}_{c r}\right)-\frac{1}{2} \delta\left(\tilde{u}_{c r}-u\right)\right) .
$$

Therein $\lambda_{e}=\lambda_{e}(u)$ according to equation (4.2), $\theta_{e}=\theta_{e}(u)$ according to equation (4.6) $)_{2}$, while the elastic contribution to the force, $F_{e}$, results in this case

$$
F_{e}=F_{e}(u)=2 b t \hat{P}_{n e o}\left(\lambda_{e}\right) \sin \theta_{e} .
$$

The evolution of both $\tilde{F}$ and $\tilde{\theta}$ while increasing the value of the externally applied displacement is illustrated in figure 6 . In particular, the pull-off force $\tilde{F}$ increases up to the critical value by following an elastic path that depends on the initial inclination $\Theta$ of the strips, and then continues to grow during delamination by exhibiting a softening with respect to the previous phase and by asymptotically tending to an upper bound for very high deformations. Such limit turns out to be independent from the initial inclination $\Theta$ and, in the particular case of unitary pre-stretch $\Lambda$ (see figure 6A), coincides with the optimal critical load $\tilde{F}_{c r}^{0}$. Contemporary, a similar behaviour can be evidenced for $\tilde{\theta}$, with the difference that, starting from the assigned initial orientation, it evolves towards a maximum critical peeling angle during the purely elastic response of the tapes and then decreases by asymptotically approaching a $\Theta$-independent inferior limit, which coincides with $\left.\tilde{\theta}_{c r}\right|_{\Theta=0}$ in absence of initial prestress in the adherent tapes part, analogously to the force. On these bases, it is possible to deduce that if one ideally progressively pulled off the free extremity of strips having an infinitely long adherent portion, beyond a certain level of deformation, this would continue to endlessly detach by keeping a constant peeling angle and by requiring a constant delamination force, in this way meeting the behaviour exhibited by the linear and symmetrical multiple peeling anchorage scheme addressed in the previous section. These results are in accordance with both laboratory observations reported in literature works (e.g. [39]) and the outcomes of preliminary experimental tests performed by the Authors, as shown in figure 7. Therein, a good agreement is revealed between experiments and theoretical predictions both in terms of forces trends and kinematics of the system, despite a certain deviation can arise as a consequence of the intrinsic differences between the actual and theoretically prescribed 

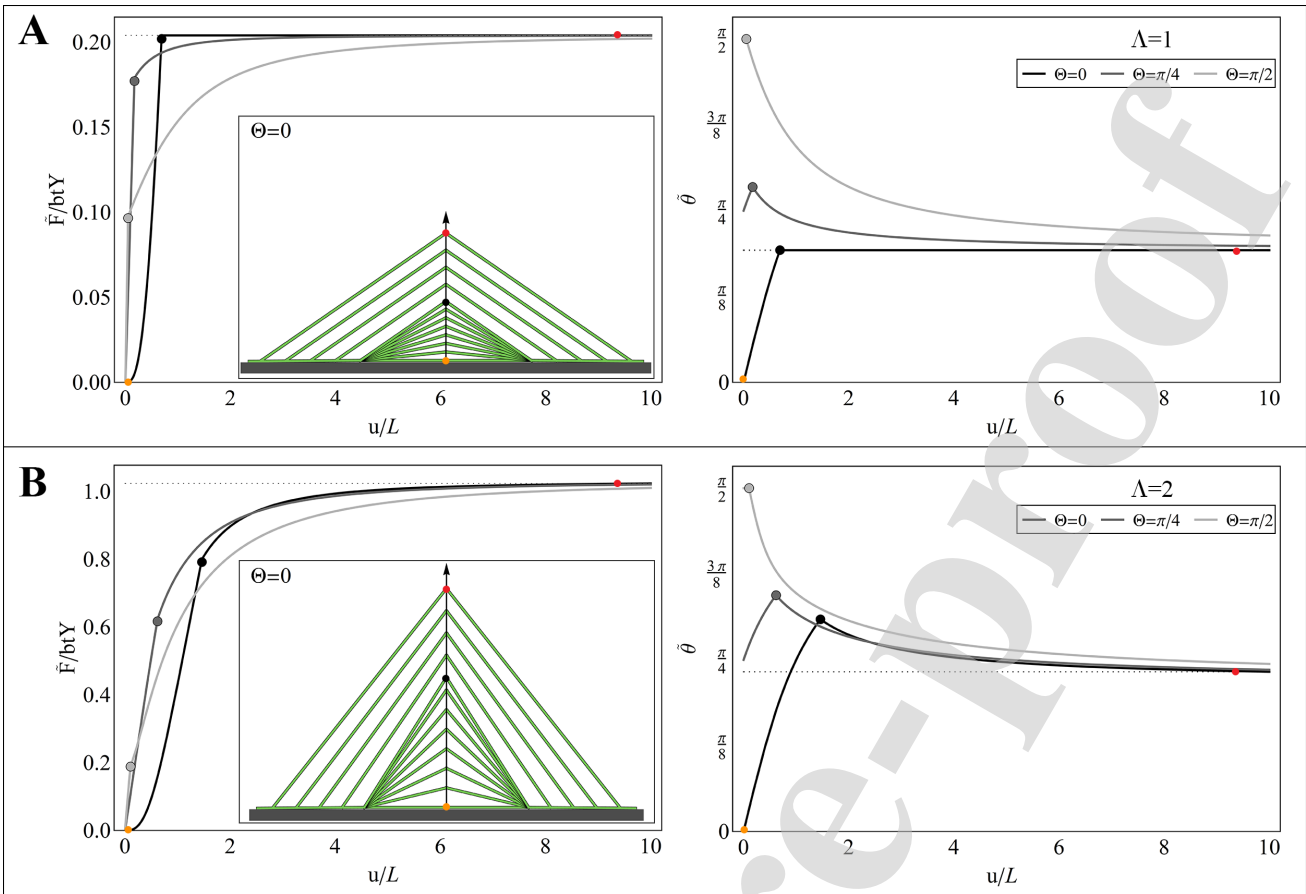

Figure 6: Trend of the (normalized) pull-off force $\tilde{F} /(b t Y)$ and of the strips' inclination angle $\tilde{\theta}$ while increasing the value of the prescribed normalized displacement $u / L$, for $\gamma=0.1$ and $\Theta=0, \pi / 4, \pi / 2$, with $\mathbf{A}) \Lambda=1$ and $\mathbf{B}) \Lambda=2$. Herein, greyscale round markers indicates critical points of peeling onset. In the insets, there are shown sketches exemplifying the evolution of the system when starting from a flat reference configuration $(\Theta=0)$.

stress-stretch responses of the hyperelastic tapes (see figure 7B) and of the experimental variability due to the physical elements' positioning and sticking operations. These can in fact lead, for example, to small imperfections in the tapes' starting inclinations, to not completely uniform adhesion levels throughout the adhering parts, as well as to not perfectly symmetrical arrangements of the tapes either at the initial configuration or during the detachment progression.

To determine an analytical expression allowing to predict the numerically found asymptotic values of the pull-off force and peeling angle in terms of the system's parameters, it is possible to follow an approximation procedure analogous to the one adopted for determining closed-form solutions for the critical values of the same quantities, namely by starting from the knowledge of these analytical limits for the constitutively linear V-shaped system. In fact, by explicitly writing down the Griffith equation in (4.18) $)_{2}$ by assuming the axial stretch within the tapes as unknown, in the limit that $d \rightarrow \infty$, it provides:

$$
\lambda^{5}+\left(3-3 \gamma \Lambda-2 \Lambda^{2}\right) \lambda^{3}-4 \lambda^{2}+2 \Lambda^{2}=0,
$$

which can be approximated to the third-order Taylor's series expansion in terms of $\lambda$ with respect to the limit value $\lambda_{\infty}^{\text {lin }}$ of the strips' axial stretch obtained in the linear case, which results from equation (3.8) as $\lambda_{\infty}^{\text {lin }}=\left.\tilde{\lambda}_{i \infty}\right|_{\left\{\gamma_{i}=\gamma, \Lambda_{i}=\Lambda\right\}}$. By following this way, a good approximation of the solution of 


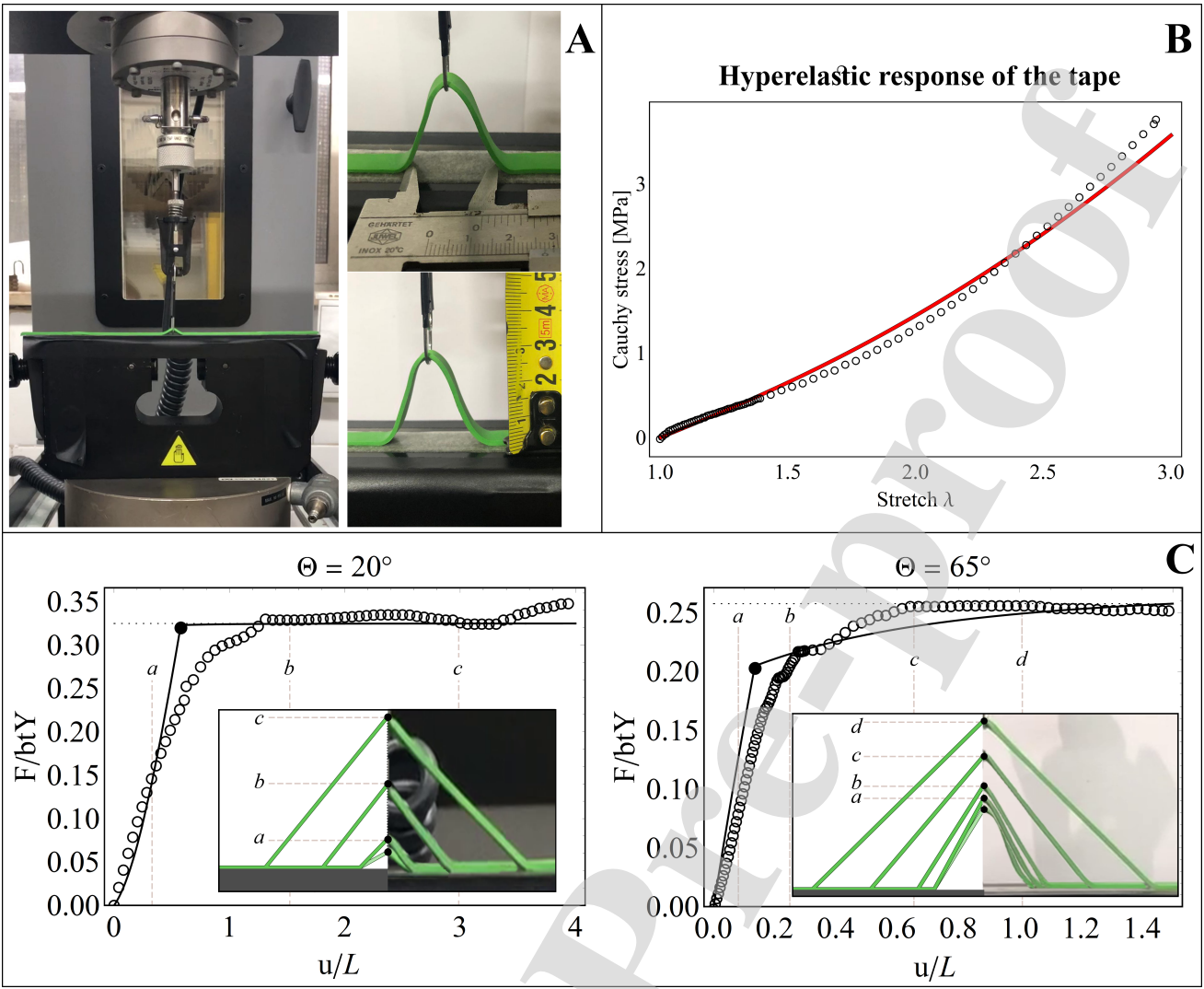

Figure 7: A) Experimental set-up used to perform double peeling tests on a V-shaped system under prescribed vertical displacement. B) Stress-stretch curve (circular points) of the hyperelastic tape used for the experiments compared with the response (solid red line) of a neo-Hookean material under uniaxial stress regime ( $Y=$ 1.24MPa). C) Comparison between experimentally observed and theoretically predicted peeling process, for different initial inclinations of the tapes, namely $\Theta=20^{\circ}$ and $\Theta=65^{\circ}$. Solid curves and circular markers identify theoretical and experimental trends of the (normalized) pull-off force $\tilde{F} /(b t Y)$, respectively, while theoretical and experimental kinematical evolutions of the system are reported in the order on the left and right sides of the insets. No pre-stretch has been imposed to the tapes in the performed test while the values of the dimensionless adhesion parameter $\gamma$ used to fit the experimental results are $\gamma=0.18$ and $\gamma=0.15$ for the two angles, respectively.

equation (4.24), say $\tilde{\lambda}_{\infty}$, is provided by:

$\tilde{\lambda}_{\infty}^{a p x}=\tilde{\lambda}_{\infty}^{a p x}(\Theta, \gamma, \Lambda)=$

$\frac{1}{3}\left\{\frac{2^{1 / 3}\left(\omega_{\infty}^{2}-3 \eta_{\infty}\right)}{\left[9 \omega_{\infty} \eta_{\infty}-2 \omega_{\infty}^{3}-27 \kappa_{\infty}+3 \sqrt{3 \eta_{\infty}^{2}\left(4 \eta_{\infty}-\omega_{\infty}^{2}\right)+6 \omega_{\infty} \kappa_{\infty}\left(2 \omega_{\infty}^{2}-9 \eta_{\infty}\right)+81 \kappa_{\infty}^{2}}\right]^{1 / 3}}\right.$
$\left.+2^{-1 / 3}\left[9 \omega_{\infty} \eta_{\infty}-2 \omega_{\infty}^{3}-27 \kappa_{\infty} a+3 \sqrt{3 \eta_{\infty}^{2}\left(4 \eta_{\infty}-\omega_{\infty}^{2}\right)+6 \omega_{\infty} \kappa_{\infty}\left(2 \omega_{\infty}^{2}-9 \eta_{\infty}\right)+81 \kappa_{\infty}^{2}}\right]^{1 / 3}-\omega_{\infty}\right\}$, 
where the coefficients $\omega_{\infty}, \eta_{\infty}$ and $\kappa_{\infty}$ are written as follows:

$$
\begin{aligned}
\omega_{\infty} & =-\frac{4\left[1+5\left(\lambda_{\infty}^{l i n}\right)^{3}\right]}{3-3 \gamma \Lambda+10\left(\lambda_{\infty}^{l i n}\right)^{2}-2 \Lambda^{2}}, \\
\eta_{\infty} & =\frac{15\left(\lambda_{\infty}^{l i n}\right)^{4}}{3-3 \gamma \Lambda+10\left(\lambda_{\infty}^{l i n}\right)^{2}-2 \Lambda^{2}}, \\
\kappa_{\infty} & =\frac{2 \Lambda^{2}-4\left(\lambda_{\infty}^{l i n}\right)^{5}}{3-3 \gamma \Lambda+10\left(\lambda_{\infty}^{l i n}\right)^{2}-2 \Lambda^{2}} .
\end{aligned}
$$

Then, the limit values of the peeling force and angle can be respectively found as follows:

$$
\begin{aligned}
& \tilde{F}_{\infty} \simeq \tilde{F}_{\infty}^{a p x}=2 b t \hat{P}_{n e o}\left(\tilde{\lambda}_{\infty}^{a p x}\right) \sin \tilde{\theta}_{\infty}^{\text {apx }}, \\
& \tilde{\theta}_{\infty} \simeq \tilde{\theta}_{\infty}^{a p x}=\arctan \left[\Lambda^{-1} \sqrt{\left(\tilde{\lambda}_{\infty}^{a p x}\right)^{2}-\Lambda^{2}}\right] .
\end{aligned}
$$

As evidenced above with reference to figure 6, expressions of such limits turn out to be independent from the initial inclination of the tapes $\Theta$ and it is not difficult to verify that, in case of unit prestretch, they coincide with the optimal critical values of the corresponding quantities, say $\tilde{F}_{0}^{a p x}$ and $\tilde{\theta}_{0}^{a p x}=\left.\tilde{\theta}_{c r}^{a p x}\right|_{\Theta=0}$. Additionally, for greater pre-stretches, the limit peeling angle is always smaller then the optimal critical one, while the inverse order relationship holds true for the pull-off forces (by way of example see figure 8).
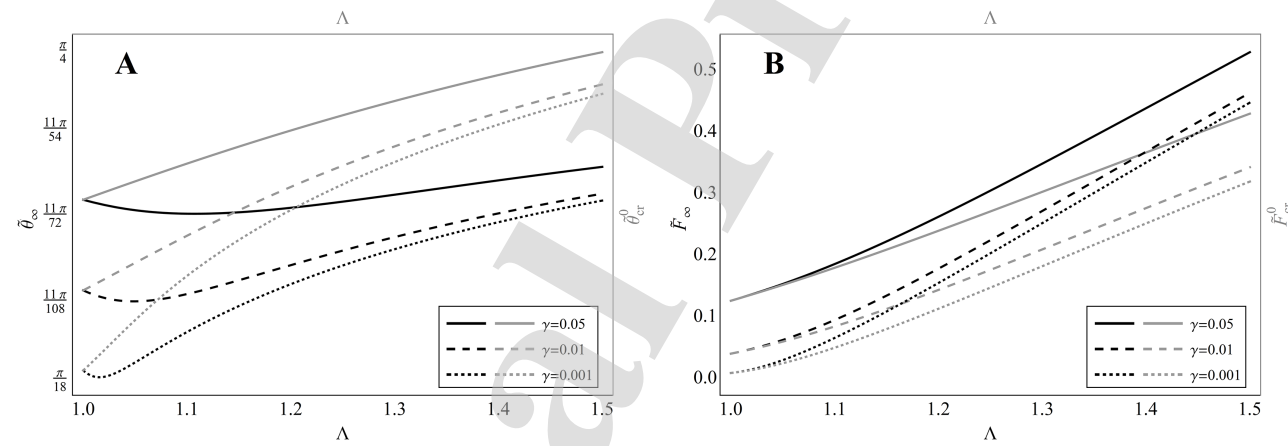

Figure 8: A) Limit value $\tilde{\theta}_{\infty} \simeq \tilde{\theta}_{\infty}^{a p x}$ to which the inclination of the V-shaped system asymptotically tends for infinitely growing pulling displacement and optimal value of the critical peeling angle $\tilde{\theta}_{c r}^{0} \simeq \tilde{\theta}_{0}^{a p x}$, plotted as functions of the pre-stretch $\Lambda$ stored within the adherent portions of the tapes, for different $\gamma$. B) Corresponding limit value $\tilde{F}_{\infty} \simeq \tilde{F}_{\infty}^{a p x}$ to which the pull-off force asymptotically tends for infinitely growing pulling displacement and optimal value of the adhesion strength $\tilde{F}_{c r}^{0} \simeq \tilde{F}_{0}^{a p x}$, plotted as functions of the pre-stretch $\Lambda$ stored within the adherent portions of the tapes, for different $\gamma$.

Finally, as done above for the purely elastic phase and for the critical event, there is the possibility to describe the evolution of the peeling process when involving tapes delamination with respect to the current configuration, in terms of relationship between the pull-off force, say $F_{d}^{\prime}$, and the peeling angle, say $\theta_{d}^{\prime}$. As a matter of fact, such a relationship coincides with the one existing between the critical values of the same quantities, since, on the bases of what already evidenced in the general section 2, equation (4.13) holds true both at the critical condition and during the quasi-static progression of the detachment. Therefore, one can found the axial pre-stretch $\lambda_{d}^{\prime}$ arising within the tapes during this latter phase as $\lambda_{d}^{\prime}\left(\theta_{d}^{\prime}\right)=\left.\lambda_{c r}^{\prime}\left(\theta_{c r}^{\prime}\right)\right|_{\theta_{c r}^{\prime}=\theta_{d}^{\prime}}$, with $\lambda_{c r}^{\prime}$ explicitly given in (4.14). Analogously, 


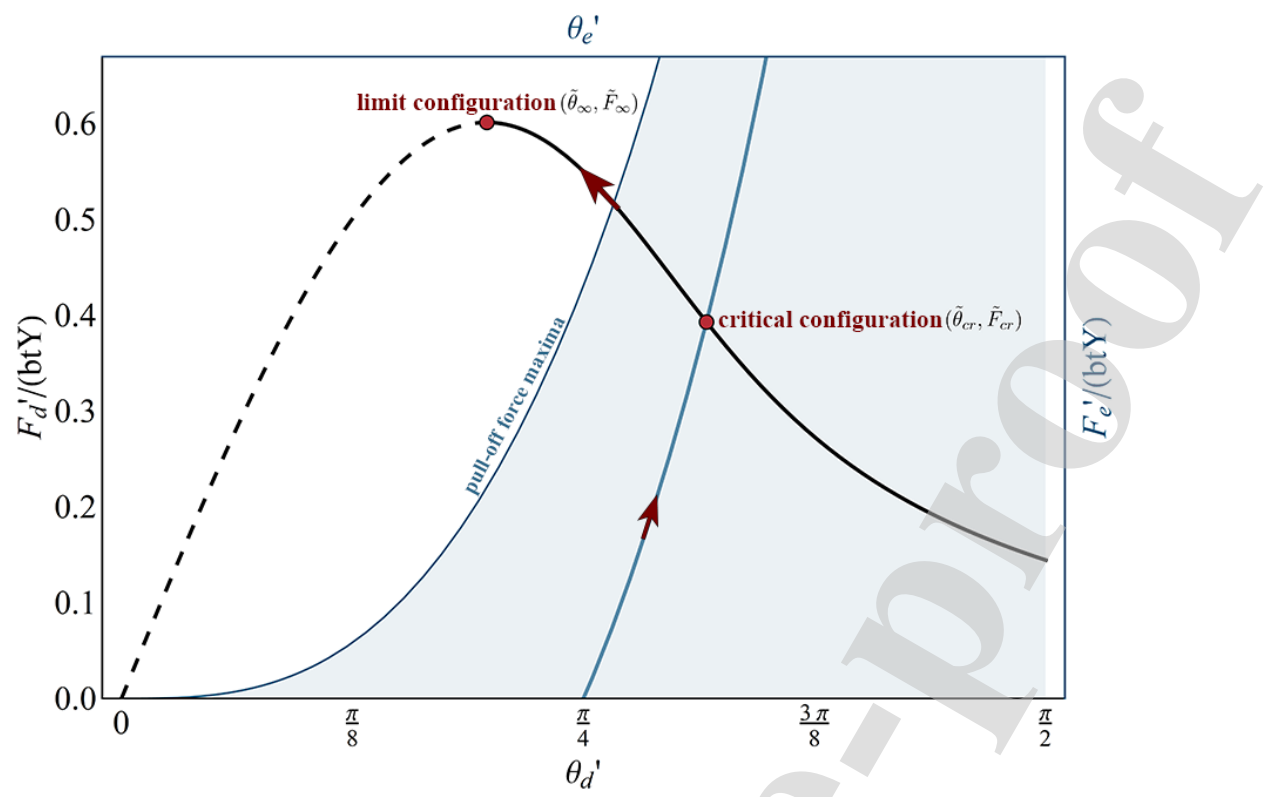

Figure 9: Normalized pull-off force versus current inclination of the tapes of the V-shaped system, both during the purely elastic response (blue curve) and during delamination (black curve), obtained for values of the system parameters chosen as: $\gamma=0.1, \Lambda=1.5$ and $\Theta=\pi / 4$. The actual path followed by the system while increasing the pulling displacement from 0 to $\infty$ for the selected parameters values, is indicated by the red arrows. Also, as explained more in detail in the main text, the dashed tract of the curve $F_{d}^{\prime} /(b t Y)$ versus $\theta_{d}^{\prime}$ make reference to theoretical configurations never experienced for by the system, while the blue domain identify points of the same curve that can actually correspond to critical configurations while considering the same values of $\gamma$ and $\Lambda$ and different initial inclinations $\Theta$. Such domain is delimited by the curve $F_{e}^{\prime} /(b t Y)$ versus $\theta_{e}^{\prime}$ obtained in case of initially flat scheme $(\Theta=0)$, which provides the adhesion strength maxima.

it is possible to obtain the associated peeling load as $F_{d}^{\prime}\left(\theta_{d}^{\prime}\right)=\left.F_{c r}^{\prime}\left(\theta_{c r}^{\prime}\right)\right|_{\theta_{c r}^{\prime}=\theta_{d}^{\prime}}, F_{c r}^{\prime}\left(\theta_{c r}^{\prime}\right)$ being given in equation (4.16), this implying that the force-inclination curves on which the system moves after the first detachment coincide with the grey-coloured lines shown in figure 5 with reference to the critical event (when considering the same values of $\gamma$ and $\Lambda$ ). In particular, analysis of its first and second derivative with respect to $\theta_{d}^{\prime}$ shows that the maximum of $F_{d}^{\prime}$ occurs in correspondence with the limit value $\tilde{\theta}_{\infty}$ to which the peeling angle asymptotically tends when ideally continuing to indefinitely pull off the system, whose approximated analytical expression $\tilde{\theta}_{\infty}^{\text {apx }}$ is given in equation $(4.27)_{2}$. As a consequence, the maximum value of $F_{d}^{\prime}$ can be well approximated by the function $\tilde{F}_{\infty}^{\text {apx }}$ in equation $(4.27)_{1}$.

In the light of these observations, the whole evolution of the peeling process can be completely represented in terms of pull-off load versus the inclination of the two tapes as done in figure 9 . Therein, the path actually followed by the system when increasingly pulling the tapes extremity, for certain fixed parameters $\gamma, \Lambda$ and $\Theta$, is indicated by the red arrows: after the purely elastic response $F_{e}^{\prime}\left(\theta_{e}^{\prime}\right)$, first detachment occurs at the critical angle $\tilde{\theta}_{c r}$ such that $F_{e}^{\prime}=F_{d}^{\prime}=\tilde{F}_{c r}$, whereupon peeling evolution proceeds by following the curve $F_{d}^{\prime}\left(\theta_{d}^{\prime}\right)$ and asymptotically tending to its maximum point $\left(\tilde{\theta}_{\infty}, \tilde{F}_{\infty}\right)$. The points that precede such maximum (dashed tract) will be hence never reached, this kind of behaviour being valid independently from the value of the system parameters. On the other hand, it is worth to underlay that, as specified with reference to figure 5, once fixed the values for $\gamma$ and $\Lambda$ and hence the curve $F_{d}^{\prime}\left(\theta_{d}^{\prime}\right)$, the critical event can in general occur at some point depending on the initial 
inclination of the tapes, strictly positioned on the right of the curve $\left.F_{e}^{\prime}\left(\theta_{e}^{\prime}\right)\right|_{\Theta=0}$ containing the critical pull-off maxima, i.e. within the blue-coloured domain. Finally, for cases of not pre-stressed adhesive tracts $(\Lambda=1)$, the critical and the limit configuration of the process coincide, the critical event also occurring at the point of maximum of the post-critical curves, in compliance with what evidenced with reference to figure $5 \mathrm{~B}$.

\section{Double peeling of a fully pre-stretched 2-tape flat configuration}

Let us consider the particular case in which the two strips constituting the V-shaped anchorage system describe an initially flat geometry (i.e. $\Theta=0$ ), thus guaranteeing maximum adhesion strength as derived above. Under this assumption, equilibrium arguments let one envisage that an additional prestretch, say $\Lambda^{*}$, can be stored in the free, i.e. non-adherent, portions of the elements, which in general can be different from the pre-stretch $\Lambda$ in the adhesive tracts. The present section is hence devoted to study the influence that such a pre-stretch could exert on the optimal value of the critical pull-off load.

By indicating as $L^{*}=\Lambda^{*} L$ the pre-stretched length of the tapes' free segments, their actual length and total stretch after the prescription of a vertical displacement $u$ can be respectively expressed as:

$$
l_{e}^{*}=\sqrt{u^{2}+\left(L^{*}\right)^{2}} \text { and } \quad \lambda_{e}^{*}=l_{e}^{*} L^{-1}
$$

with reference to the purely elastic response of the system, and as

$$
l_{d}^{*}=\sqrt{u^{2}+\left(L^{*}+d\right)^{2}} \text { and } \lambda_{d}^{*}=l_{d}^{*} L_{d}^{-1},
$$

in case of detachment of a tract $d$. Then, the critical displacement $u_{c r}^{*}$, inducing the onset of the detachment process in the present case, has to be found by requiring the observance of the equation:

$$
-\left.\frac{\partial \Pi_{d}^{*}}{\partial d}\right|_{d \rightarrow 0}=b R, \quad \Pi_{d}^{*}=b t L_{d} \hat{\Psi}_{n e o}\left(\lambda_{d}^{*}\right)
$$

which, by performing algebraic manipulations, results in

$$
\left(\lambda_{e}^{*}\right)^{5}+\left(3-3 \gamma \Lambda-2 \Lambda \Lambda^{*}\right)\left(\lambda_{e}^{*}\right)^{3}-4\left(\lambda_{e}^{*}\right)^{2}+2 \Lambda \Lambda^{*}=0
$$

By resorting to numerical solution, such equation hence leads to obtain $u_{c r}^{*}$ and the related critical peeling force $F_{c r}^{*}$, that is:

$$
F_{c r}^{*}=\frac{2}{3} b t Y \frac{u_{c r}^{*}}{L}\left[1-\left(\left(\frac{u_{c r}^{*}}{L}\right)^{2}+\left(\Lambda^{*}\right)^{2}\right)^{-3 / 2}\right],
$$

as functions of $\Lambda^{*}$ and $\Lambda$, once fixed the geometrical, elastic and adhesive parameters of the model. The results evidence that the storing of a pre-stretch within the free parts of the tapes can actually affect the optimal adhesion strength, as shown in figure 10. In particular, in figure 10A, all the possible critical pull-off states of the flat and pre-stretched anchorage system are schematized in the phase plane defined by the variables $\Lambda$ and $\Lambda^{*}$. Therein, two complementary domains can be immediately recognized, identified as compatibility and incompatibility regions. The former comprises the plane points corresponding to pairs of pre-stretches' values such that equation (5.4) admits real solutions, thus providing non-negative critical peeling forces $F_{c r}^{*}$ according to the expression in (5.5). Within this region, the competition between the values of the two pre-stretches determines a non-monotonic 

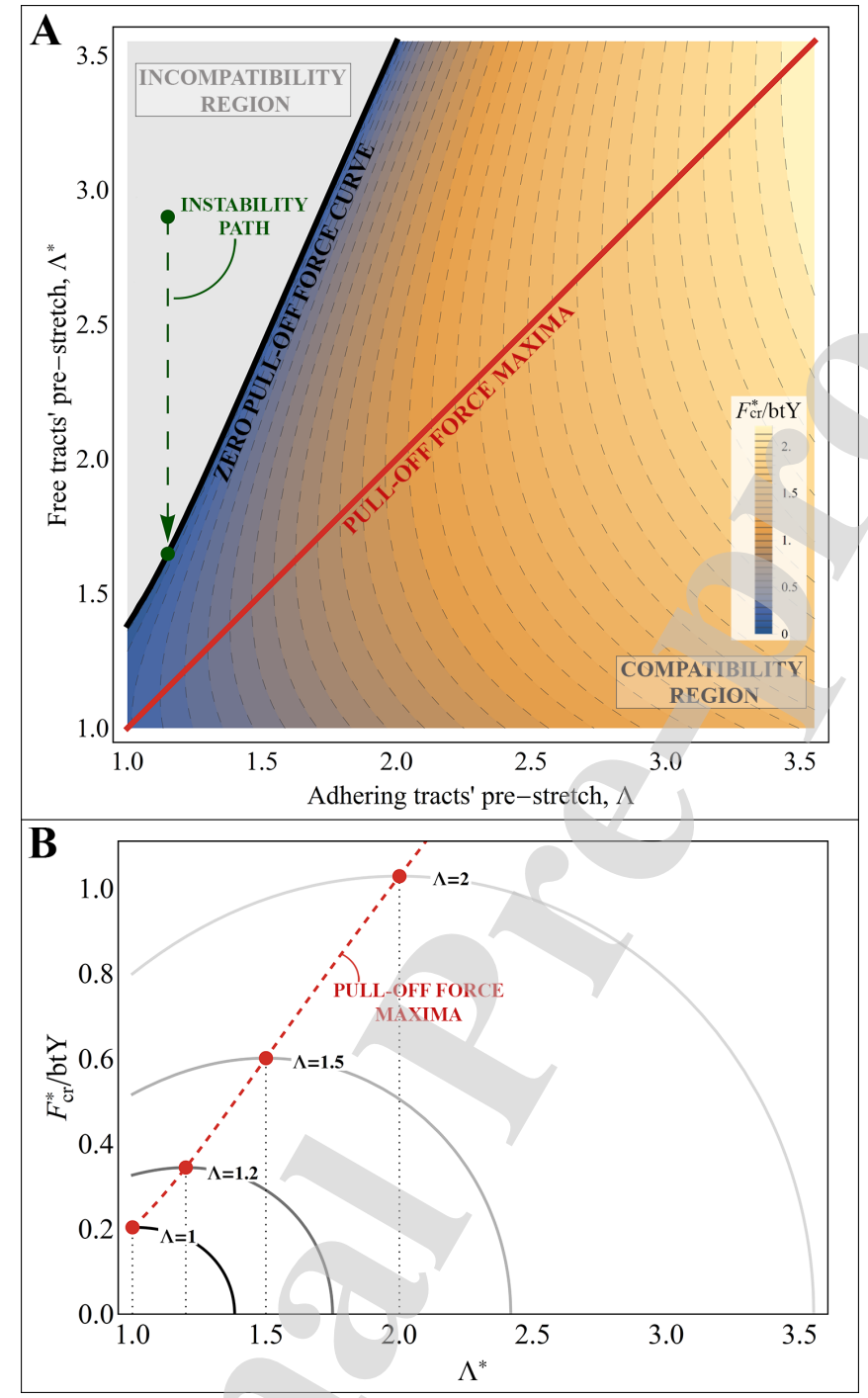

Figure 10: A) A schematic representation of the possible critical pull-off states for a flat and pre-stretched 2-tape adhesive system, in the phase plane identified by the pre-stretches $\Lambda$ and $\Lambda^{*}$, in the adhering and free tracts of the tapes respectively. In the so-called compatibility region (described in detail in the main text) a density plot of the normalized critical pull-off force $F_{c r}^{*} /(b t Y)$ is also shown. B) Non-monotonic trend of $F_{c r}^{*} /(b t Y)$ while increasing the pre-stretch $\Lambda^{*}$, for fixed values of $\Lambda$. Herein, the curve corresponding to the pull-off force maxima, occurring when $\Lambda^{*}=\Lambda$, is also evidenced. All the plots have been obtained for a value of the dimensionless adhesion parameter $\gamma$ fixed at 0.1 .

trend of $F_{c r}^{*}$ when increasing $\Lambda^{*}$ with respect to the unit, for any selected $\Lambda$ (see both figures $10 \mathrm{~A}$ and 10B). Importantly, the maxima of the critical pull-off load are found to rely on the bisector of the phase plane and hence occur when the pre-stretch in the free tracts equates that of the adhering portions, i.e.:

$$
\left.\frac{\partial F_{c r}^{*}}{\partial \Lambda^{*}}\right|_{\Lambda^{*}=\Lambda}=0,\left.\frac{\partial^{2} F_{c r}^{*}}{\partial\left(\Lambda^{*}\right)^{2}}\right|_{\Lambda^{*}=\Lambda}<0 .
$$


Additionally, a zero pull-off force curve can be identified, at the boundary with the incompatibility region, as the locus of points such that a vanishing load (or displacement), applied to the pre-stretched flat configuration, is sufficient to initiate the detachment process. The implicit expression of this curve can be easily determined by assuming $u=0$ in equation (5.4). On the other hand, the incompatibility region is constituted by the pairs of strips' pre-stretches for which the energy balance in equation (5.3) can never be satisfied, since, for any fixed $\Lambda$ of the adhering segments, the energy stored through a pre-stretch $\Lambda^{*}$ over the zero pull-off force curve would be itself greater than that required to trigger the delamination by means of an infinitesimal applied load. This means that it is not possible to conceive a stable pre-stretched configuration of the adhesive tapes' system for assigned couples of $\Lambda$ and $\Lambda^{*}$ in that region of the plane, whence its identification as incompatibility region. In fact, by way of example, one can likely envisage that, by pre-stretching the tapes in a way to fall in the incompatibility domain, the system would naturally evolve by partially detaching before the application of any external load, in this way relaxing the pre-stretch in the free tracts of the strips and moving itself along an instability path towards the closest compatible configuration, which coincides with the point of the zero pull-off force curve that preserves the pre-stretch in the adhering portions. It is worth noting that, in figure 10 , the adhesion parameter $\gamma$ is fixed to 0.1 , different choices not influencing the quality of the described results.

\section{Remarks on asymmetrical peeling modes}

In this section, the possibility that peeling of V-shaped systems under prescribed vertical displacement occurs by following asymmetrical configurations is briefly analyzed with the purpose of establishing if deviations from the above-considered symmetrical shape could eventually lead to earlier or energetically more convenient detachment. To investigate such a question, it is here envisaged that, by selecting a certain actual configuration in which the two tapes are overall detached for a portion $2 d$, this length is distributed between the two sides of the structure according to the fractions $\rho$ and $1-\rho$, as in figure $2 \mathrm{C}$. In particular, the parameter $\rho$ is assumed to range within the interval $[0,1]$, in this way recovering the symmetry condition when $\rho=1 / 2$. Also, in compliance with the asymmetry of the detachment and of the related deformation of the tapes, a further horizontal displacement $u_{h}$ might occur at the common vertex. Hence, giving as follows the geometrical relations that describe the kinematics for this specific case, i.e.

$$
\begin{aligned}
& L_{\rho}=L+2 \rho d \Lambda^{-1}, \quad L_{1-\rho}=L+2(1-\rho) d \Lambda^{-1}, \\
& l_{\rho}=\sqrt{(L \sin \Theta+u)^{2}+\left(L \cos \Theta+u_{h}+2 \rho d\right)^{2}}, \\
& l_{1-\rho}=\sqrt{(L \sin \Theta+u)^{2}+\left[L \cos \Theta-u_{h}+2(1-\rho) d\right]^{2}}, \\
& \lambda_{\rho}=l_{\rho} L_{\rho}^{-1}, \quad \lambda_{1-\rho}=l_{1-\rho} L_{1-\rho}^{-1},
\end{aligned}
$$

the total potential energy of the system can be properly written as

$$
\Pi=\Pi_{\rho}+\Pi_{1-\rho}, \quad \text { where } \quad \Pi_{\rho, 1-\rho}=\frac{Y b t L_{\rho, 1-\rho}\left(2-3 \lambda_{\rho, 1-\rho}+\lambda_{\rho, 1-\rho}^{3}\right)}{6 \lambda_{\rho, 1-\rho}}
$$

are the elastic energies of the two strips, respectively, under the assumption of neo-Hookean hyperelasticity and incompressibility.

On these bases, requirement of equilibrium leads to the imposition of the stationary energy condition

$$
\frac{\partial \Pi}{\partial u_{h}}=0
$$


whose resolution provides the horizontal displacement at equilibrium as a function of the prescribed vertical displacement $u$, of the delamination length $d$ and of the asymmetry ratio $\rho$, once fixed all the other geometrical and constitutive parameters, namely $\tilde{u}_{h}=\tilde{u}_{h}(u, d, \rho)$. Then, the Griffith criterion can be formulated in the present case as

$$
-\frac{\partial}{\partial d}\left(\left.\Pi\right|_{u_{h}=\tilde{u}_{h}}\right)=2 R b
$$

whence the delamination function, say $\tilde{d}$, describing the quasi-static evolution of $d$, can be derived for all $u$ and $\rho$.

To the aim of finding closed-form solutions for the problem at hand, the system constituted by the coupling of the equations (6.3) and (6.4) has been solved by replacing the energy $\Pi$ with its secondorder Maclaurin series expansion with respect to $u_{h}$ and $d$, here indicated as say $\Pi_{a p x}$. This hence allowed to study analytically the evolution of the system in the first phase of the tapes detachment by starting from the purely elastic symmetrical configuration preceding the critical pull-off condition, where both $u_{h}$ and $d$ vanish. By following this way, it has been possible to verify that the critical displacement $u_{c r}$ and, hence, the critical force $F_{c r}$ do not depend on the asymmetry ratio $\rho$, they $d e$ facto coinciding with the ones found for the symmetrical delamination. Additionally, by numerically analyzing the trend of the total (approximated) energy of the system in solution, defined as

$$
E(u ; \rho)=\left.\Pi_{a p x}\right|_{\left\{d=\tilde{d}(u ; \rho), u_{h}=\tilde{u}_{h}(u, \tilde{d}(u ; \rho) ; \rho)\right\}}+2 b R \tilde{d}(u ; \rho),
$$

while varying $\rho$ within the range $[0,1]$, it has been possible to observe that, for any reference configuration, chosen material parameters, prescribed pre-stretch and imposed vertical displacement (greater than the critical one), symmetrically detached arrangements actually correspond to the minimum energy ones.

These results lead to the conclusion that, at least in principle, a perfect V-shaped anchorage scheme of tapes could equivalently initiate to detach according to a symmetrical or asymmetrical mode under the same critical value of imposed vertical displacement (or force). As a consequence, if one dealt with a system made of two strips adhering to a rigid substrate for an infinitesimally extended tract, one could indifferently detect a full (contemporary) symmetrical detachment of them or two consecutive peeling events at the extremities. However, if the adhesive portions of the elastic strips had a finite length, one should always observe a symmetrical progression of the peeling process.

\section{Conclusion}

In the present work, the theory of multiple peeling has been extended by primarily including large deformations and nonlinear hyperelasticity of the adhesive elements. Starting from a general framework, in which a number of converging tapes partially adhering over a flat rigid substrate is considered, the peeling of a symmetrical system of constitutively linear strips under prescribed vertical displacement has been analytically studied by borrowing the Griffith criterion from the fracture mechanics theory. Then, on these bases, an (incompressible) neo-Hookean hyperelastic law has been adopted to model the double peeling process of a $\mathrm{V}$-shaped anchorage scheme comprising two strips undergoing finite deformations. By following this way, the critical values of the pull-off displacement and force inducing the first peeling event have been derived as functions of the geometrical, constitutive and adhesive properties of the system and of the pre-stretch stored within the adhering strips tracts, by also gaining some closed-form solutions. We have found that the reference configuration providing optimal adhesion, namely the one requiring maximum critical pull-off force to initiate detaching, always occurs when the two strips are in an initially flat configuration with vanishing relative inclination, and that the pre-stretch of the adhesive portions contributes to delay the peeling onset by raising the pulling force 
that can be sustained before detachment starts for fixed values of the other parameters. Additionally, from a comparison between the outcomes resulting from the presented model and those obtained by assuming linear elastic elements, we have observed a substantial discrepancy in terms of critical load, which results particularly significant for geometries of the system close to the optimal one and increases with the pre-stretch within the tapes. This de facto corroborates the need of introducing nonlinear hyperelasticy to the aim of modelling peeling of highly deformable tapes anchorages. Analysis of the system evolution under the hypothesis of endless adherent tracts has then led to the finding that both the pull-off force and the peeling angle stabilize on constant asymptotic limits when progressively increasing the applied displacement over the critical value. Importantly, such limits turn out to be independent from the initial inclination of the tapes and respectively coincide with the optimal critical pull-off force and critical peeling angle in case of not pre-stressed condition. The obtained theoretical predictions are then compared with results provided by experimental tests. Furthermore, the effects of a pre-stretch stored in the free tracts of the tapes -admissible in the case of an initially flat (hence optimal) configuration- have been analyzed, in this way revealing a possible additional increase of the critical pull-off force, which results to be maximum when the values of the pre-stretches in the adhering and non-adhering portions of the anchorage system coincide. Finally, asymmetrical detachment progression of the V-shaped structure has been investigated, in this way showing that, despite the critical force is not influenced by the peeling mode, any asymmetrically detached configuration would be energetically less convenient than a symmetrical one at the same applied vertical displacement. By starting from these results, other biomechanical problems could be addressed ex-

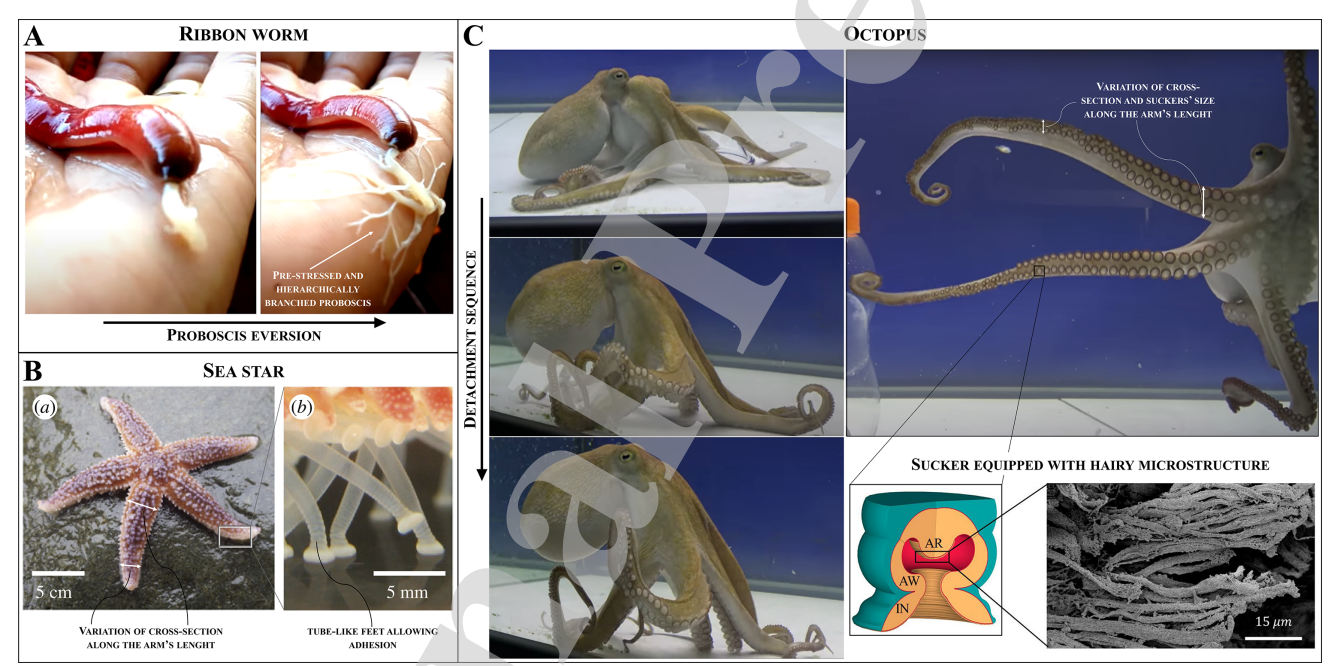

Figure 11: A) A specimen of Nemertea, also known as ribbon worms or proboscis worms, whose branching proboscis can be everted through muscle contraction for defence or motion aims as well as for the capture of small invertebrates (source: Science Channel). B) Image of a common sea star Asterias Rubens, with a close-up on the tube feet lining the ventral surface allowing adhesion and motion, adapted from the work by Heydari et al. [53]. Variation of arms' cross-section is highlighted. C) On the left, a specimen of Octopus Vulgaris detaching from a surface by reproducing a sort of V-shaped multiple peeling process (source: Octolab.tv videos). On the right, a focus on the non-homogeneous distribution of cross-sections' and suckers' size along the arms' length, with a zoom on the suckers' hairy microstructure (adapted from the work by Greco et al. [54].)

ploiting the proposed nonlinear peeling model. Since pre-tension has emerged as a factor able to significantly influence the adhesion strength of adherent systems as well as the values of the pull-off force required for carrying out the whole peeling process, the modelling of pre-stretched hyperelastic tapes could for instance help the investigation of optimization strategies employed by natural adhesive 
structures for tuning (principally maximizing) their bonding/debonding capabilities. In this regard, an example is provided by geckos' pads, in which the pre-tension induced by the stretching of the spatulae while establishing contact, seems to contribute to explain how extraordinary attachment and rapid and controllable detachment are achieved [34,55]. Tuning of pre-stress is also employed by cells as optimization mechanism to modulate the local and overall adhesion strength in a way to functionally promote stable adherent configurations and to facilitate detachment prodromal to migration. Furthermore, new insights into the effects of pre-stretch regulation could contribute to improve or redesign artificial adhesion systems, one possible direct application being related to dry adhesive medical skin patches $[14,15]$. On the other hand, it is worth noticing that, by introducing non-homogeneities, the proposed nonlinear peeling model would allow to take into account non-homogeneous geometrical, material or adhesive properties of the tapes, including specific scaling laws and hierarchical configurations of the anchorage structures across multiple length scales. This approach would help the study of the optimized peeling mechanics of multi-level hairy terminals observed in spiders and geckos and the understanding of the mechanisms ruling extreme adhesive properties of rarely met animals, such as Nemertea, that also show branching adhesive terminals in which strong nonlinearities and pre-tension seem to be diriment (see figure 11A). Cooperation between non-homogeneities and nonlinearity in multiple peeling models could be additionally crucial to analyse some optimization strategies highlighted by the arms of sea stars and octopuses (see figure 11B,C). Morphology and functionality of octopus' suckers have been in fact recently investigated $[54,56]$ and peculiar scaling laws could be discovered to maximize the critical pull-off load, to enhance overall toughness and to promote autotomy (i.e. self-amputation) all these "object functions" being at the basis of self-defense behaviours of these animals.

It is felt that the nonlinear multiple peeling model proposed here might be helpfully adopted to gain new insights into the mechanisms governing the enhanced adhesion capabilities of peculiar biomechanical anchorage systems, as well as to guide the design of novel artificial (e.g. biomimetic) adhesive devices, in which geometry, hyperelasticity and pre-stress can be all exploited and properly dosed to modulate and optimize the adhesion degree for possible applications in soft robotics and materials engineering.

Acknowledgments MF, SP and NP gratefully acknowledge the support of the Italian Ministry of Education, University and Research (MIUR) through the grant PRIN-20177TTP3S. MF and NP also acknowledge the support of the MIUR through the grant PON-ARS01_01384. NP is also supported by the European Commission H2020 FET Proactive "Neurofibres" grant no. 732344 and FET Open "Boheme" grant no. 863179, as well as by the MIUR under the "Departments of Excellence" grant L.232/2016. ARC acknowledges the support of the grant PON-AIM1849854-1.

\section{References}

[1] D. Labonte and W. Federle, "Biomechanics of shear-sensitive adhesion in climbing animals: peeling, pre-tension and sliding-induced changes in interface strength," Journal of The Royal Society Interface, vol. 13, no. 122, p. 20160373, 2016.

[2] K. Autumn, Y. A. Liang, S. T. Hsieh, W. Zesch, W. P. Chan, T. W. Kenny, R. Fearing, and R. J. Full, "Adhesive force of a single gecko foot-hair," Nature, vol. 405, pp. 681-685, jun 2000.

[3] K. Autumn, "Frictional adhesion: a new angle on gecko attachment," Journal of Experimental Biology, vol. 209, pp. 3569-3579, sep 2006. 
[4] E. Arzt, S. Gorb, and R. Spolenak, "From micro to nano contacts in biological attachment devices," Proceedings of the National Academy of Sciences, vol. 100, pp. 10603-10606, sep 2003.

[5] M. Varenberg, N. M. Pugno, and S. N. Gorb, "Spatulate structures in biological fibrillar adhesion," Soft Matter, vol. 6, no. 14, p. 3269, 2010.

[6] N. Pugno, E. Lepore, S. Toscano, and F. Pugno, "Normal adhesive force-displacement curves of living geckos," The Journal of Adhesion, vol. 87, no. 11, pp. 1059-1072, 2011.

[7] H. Yao and H. Gao, "Mechanics of robust and releasable adhesion in biology: Bottom-up designed hierarchical structures of gecko," Journal of the Mechanics and Physics of Solids, vol. 54, pp. 1120-1146, jun 2006.

[8] A. Pantano, N. M. Pugno, and S. N. Gorb, "Numerical simulations demonstrate that the double tapering of the spatualae of lizards and insects maximize both detachment resistance and stability," International Journal of Fracture, vol. 171, pp. 169-175, apr 2011.

[9] S. Flenner, C. F. Schaber, I. Krasnov, H. Stieglitz, M. Rosenthal, M. Burghammer, S. N. Gorb, and M. Müller, "Multiple mechanical gradients are responsible for the strong adhesion of spider attachment hair," Advanced Materials, vol. 32, no. 37, p. 2002758, 2020.

[10] C. F. Schaber, S. Flenner, A. Glisovic, I. Krasnov, M. Rosenthal, H. Stieglitz, C. Krywka, M. Burghammer, M. Müller, and S. N. Gorb, "Hierarchical architecture of spider attachment setae reconstructed from scanning nanofocus x-ray diffraction data," Journal of The Royal Society Interface, vol. 16, no. 150, p. 20180692, 2019

[11] H. Gao, X. Wang, H. Yao, S. Gorb, and E. Arzt, "Mechanics of hierarchical adhesion structures of geckos," Mechanics of Materials, vol. 37, pp. 275-285, feb 2005.

[12] F. Bosia, S. Colella, V. Mattoli, B. Mazzolai, and N. M. Pugno, "Hierarchical multiple peeling simulations," RSC Adv., vol. 4, pp. 25447-25452, 2014.

[13] K. Autumn, M. Sitti, Y. A. Liang, A. M. Peattie, W. R. Hansen, S. Sponberg, T. W. Kenny, R. Fearing, J. N. Israelachvili, and R. J. Full, "Evidence for van der waals adhesion in gecko setae," Proceedings of the National Academy of Sciences, vol. 99, pp. 12252-12256, aug 2002.

[14] M. K. Kwak, H.-E. Jeong, and K. Y. Suh, "Rational design and enhanced biocompatibility of a dry adhesive medical skin patch," Advanced Materials, vol. 23, pp. 3949-3953, jul 2011.

[15] S. Baik, H. J. Lee, D. W. Kim, J. W. Kim, Y. Lee, and C. Pang, "Bioinspired adhesive architectures: From skin patch to integrated bioelectronics," Advanced Materials, vol. 31, no. 34, p. $1803309,2019$.

[16] J. Y. Chung and M. K. Chaudhury, "Roles of discontinuities in bio-inspired adhesive pads," Journal of The Royal Society Interface, vol. 2, pp. 55-61, mar 2005.

[17] A. K. Geim, S. V. Dubonos, I. V. Grigorieva, K. S. Novoselov, A. A. Zhukov, and S. Y. Shapoval, "Microfabricated adhesive mimicking gecko foot-hair," Nature Materials, vol. 2, pp. 461-463, jun 2003.

[18] C. Greiner, A. del Campo, and E. Arzt, "Adhesion of bioinspired micropatterned surfaces: effects of pillar radius, aspect ratio, and preload," Langmuir, vol. 23, pp. 3495-3502, mar 2007. 
[19] R. Spolenak, S. Gorb, H. Gao, and E. Arzt, "Effects of contact shape on the scaling of biological attachments," Proceedings of the Royal Society A: Mathematical, Physical and Engineering Sciences, vol. 461, pp. 305-319, feb 2005.

[20] L. Heepe and S. N. Gorb, "Biologically inspired mushroom-shaped adhesive microstructures," Annual Review of Materials Research, vol. 44, pp. 173-203, jul 2014.

[21] W. Federle and D. Labonte, "Dynamic biological adhesion: mechanisms for controlling attachment during locomotion," Philosophical Transactions of the Royal Society B: Biological Sciences, vol. 374, no. 1784, p. 20190199, 2019.

[22] L. Afferrante, G. Carbone, G. Demelio, and N. Pugno, "Adhesion of elastic thin films: Double peeling of tapes versus axisymmetric peeling of membranes," Tribology Letters, vol. 52, pp. 439-447, oct 2013.

[23] M. Fraldi, S. Palumbo, A. Carotenuto, A. Cutolo, L. Deseri, and N. Pugno, "Buckling soft tensegrities: Fickle elasticity and configurational switching in living cells," Journal of the Mechanics and Physics of Solids, vol. 124, pp. 299-324, mar 2019.

[24] M. Fraldi, A. Cugno, A. R. Carotenuto, A. Cutolo, N. M. Pugno, and L. Deseri, "Small-on-large fractional derivative-based single-cell model incorporating cytoskeleton prestretch," Journal of Engineering Mechanics, 2017.

[25] G. Alotta, E. Bologna, M. D. Giuseppe, M. Zingales, R. Dimitri, F. P. Pinnola, and G. Zavarise, "A non-local mode-i cohesive model for ascending thoracic aorta dissections (ATAD)," in 2018 IEEE 4th International Forum on Research and Technology for Society and Industry (RTSI), IEEE, sep 2018.

[26] F. Nappi, A. R. Carotenuto, A. Cutolo, P. Fouret, C. Acar, J. C. Chachques, and M. Fraldi, "Compliance mismatch and compressive wall stresses drive anomalous remodelling of pulmonary trunks reinforced with dacron grafts," Journal of the mechanical behavior of biomedical materials, vol. 63, pp. 287-302, 2016.

[27] O. Tricinci, E. V. Eason, C. Filippeschi, A. Mondini, B. Mazzolai, N. M. Pugno, M. R. Cutkosky, F. Greco, and V. Mattoli, “Approximating gecko setae via direct laser lithography," Smart Materials and Structures, vol. 27, p. 075009, may 2018.

[28] N. M. Pugno, “The theory of multiple peeling," International Journal of Fracture, vol. 171, pp. 185-193, oct 2011.

[29] K. Kendall, “Thin-film peeling-the elastic term,” Journal of Physics D: Applied Physics, vol. 8, pp. 1449-1452, sep 1975

[30] K. Kendall, "The adhesion and surface energy of elastic solids," Journal of Physics D: Applied Physics, vol. 4, pp. 1186-1195, aug 1971.

[31] D. H. Kaelble, "Theory and analysis of peel adhesion: Bond stresses and distributions," Transactions of the Society of Rheology, vol. 4, no. 1, pp. 45-73, 1960.

[32] J. J. Bikerman, "Theory of peeling through a hookean solid," Journal of Applied Physics, vol. 28, no. 12 , pp. 1484-1485, 1957.

[33] B. Chen, P. Wu, and H. Gao, "Hierarchical modelling of attachment and detachment mechanisms of gecko toe adhesion," Proceedings of the Royal Society A: Mathematical, Physical and Engineering Sciences, vol. 464, pp. 1639-1652, jun 2008. 
[34] B. Chen, P. Wu, and H. Gao, "Pre-tension generates strongly reversible adhesion of a spatula pad on substrate," Journal of The Royal Society Interface, vol. 6, pp. 529-537, jun 2009.

[35] L. Brely, F. Bosia, and N. M. Pugno, "Numerical implementation of multiple peeling theory and its application to spider web anchorages," Interface Focus, vol. 5, pp. 20140051-20140051, dec 2014.

[36] N. Menga, L. Afferrante, N. Pugno, and G. Carbone, "The multiple v-shaped double peeling of elastic thin films from elastic soft substrates," Journal of the Mechanics and Physics of Solids, vol. 113, pp. 56-64, apr 2018.

[37] D. Misseroni, L. Afferrante, G. Carbone, and N. M. Pugno, "Non-linear double-peeling: Experimental vs. theoretical predictions," The Journal of Adhesion, vol. 94, pp. 46-57, feb 2017.

[38] C. Putignano, L. Afferrante, L. Mangialardi, and G. Carbone, "Equilibrium states and stability of pre-tensioned adhesive tapes," Beilstein Journal of Nanotechnology, vol. 5, pp. 1725-1731, oct 2014.

[39] L. Heepe, S. Raguseo, and S. N. Gorb, "An experimental study of double-peeling mechanism inspired by biological adhesive systems," Applied Physics A, vol. 123, jan 2017.

[40] C. Creton and M. Ciccotti, "Fracture and adhesion of soft materials: a review," Reports on Progress in Physics, vol. 79, p. 046601, mar 2016.

[41] Z. Peng and S. Chen, "Effect of bending stiffness on the peeling behavior of an elastic thin film on a rigid substrate," Physical Review E, vol. 91, apr 2015.

[42] C. Majidi, "Shear adhesion between an elastica and a rigid flat surface," Mechanics Research Communications, vol. 36, pp. 369-372, apr 2009.

[43] R. A. Sauer, "The peeling behavior of thin films with finite bending stiffness and the implications on gecko adhesion," The Journal of Adhesion, vol. 87, pp. 624-643, jul 2011.

[44] A. Molinari and G. Ravichandran, "Peeling of elastic tapes: Effects of large deformations, prestraining, and of a peel-zone model," The Journal of Adhesion, vol. 84, no. 12, pp. 961-995, 2008.

[45] M. R. Begley, R. R. Collino, J. N. Israelachvili, and R. M. McMeeking, "Peeling of a tape with large deformations and frictional sliding," Journal of the Mechanics and Physics of Solids, vol. 61 , no. 5, pp. $1265-1279,2013$.

[46] Z. Peng and S. Chen, "Peeling behavior of a thin-film on a corrugated surface," International Journal of Solids and Structures, vol. 60-61, pp. 60-65, may 2015.

[47] A. Ozer, "The effect of substrate roughness on tape peeling," International Journal of Adhesion and Adhesives, vol. 62, pp. 14-17, oct 2015.

[48] L. Brely, F. Bosia, S. Palumbo, M. Fraldi, A. Dhinojwala, and N. M. Pugno, "Competition between delamination and tearing in multiple peeling problems," Journal of The Royal Society Interface, vol. 16, no. 160, p. 20190388, 2019.

[49] G. A. Maugin, The thermomechanics of plasticity and fracture, vol. 7. Cambridge University Press, 1992.

[50] G. A. Holzapfel, Nonlinear Solid Mechanics: A Continuum Approach for Engineering. Wiley, 2000. 
[51] S. Palumbo, A. R. Carotenuto, A. Cutolo, L. Deseri, and M. Fraldi, "Nonlinear elasticity and buckling in the simplest soft-strut tensegrity paradigm," International Journal of Non-Linear Mechanics, vol. 106, pp. 80-88, nov 2018.

[52] I. Wolfram Research, Mathematica. Wolfram Research, Inc., 2015.

[53] S. Heydari, A. Johnson, O. Ellers, M. J. McHenry, and E. Kanso, "Sea star inspired crawling and bouncing," Journal of The Royal Society Interface, vol. 17, no. 162, p. 20190700, 2020.

[54] G. Greco, F. Bosia, F. Tramacere, B. Mazzolai, and N. M. Pugno, "The role of hairs in the adhesion of octopus suckers: a hierarchical peeling approach," Bioinspiration \& Biomimetics, vol. 15, p. 035006, mar 2020.

[55] Q. H. Cheng, B. Chen, H. J. Gao, and Y. W. Zhang, "Sliding-induced non-uniform pre-tension governs robust and reversible adhesion: a revisit of adhesion mechanisms of geckos," Journal of The Royal Society Interface, vol. 9, no. 67, pp. 283-291, 2012.

[56] F. Tramacere, L. Beccai, M. Kuba, A. Gozzi, A. Bifone, and B. Mazzolai, "The morphology and adhesion mechanism of octopus vulgaris suckers," PLOS ONE, vol. 8, pp. 1-7, 062013. 
Trento/Napoli - Italy, July $15^{\text {th }} 2020$

To the attention of the

Editors-in-Chief: Professors K. J. Hsia, J. A. Rogers, Z. Suo

Extreme Mechanics Letters

Editors of VSI Espinosa Prager Medal: Professors F. Barthelat, Y. Zhu, P. Zavattieri

Dear Editors,

with reference to the paper entitled

\section{GENERALIZED MULTIPLE PEELING THEORY UPLOADING HYPERELASTICITY AND PRE-STRESS}

by M. Fraldi, S. Palumbo, A.R. Carotenuto, A. Cutolo, and N. Pugno

submitted for possible publication on Extreme Mechanics Letters - VSI: Espinosa Prager Medal,

all the authors declare to have no conflict of interest.

Sincerely

Nicola Pugno ${ }^{1}$ and Massimiliano Fraldi ${ }^{2}$

Professor of Mechanics (E-mail: nicola.pugno@unitn.it)

Department of Civil, Environmental and Mechanical Engineering

University of Trento, Trento - ITALY

2 Professor of Continum and Tissue Mechanics (E-mail: fraldi@unina.it)

Department of Structures for Engineering and Architecture

Polytechnic School - College of Engineering, University of Napoli Federico II, Napoli-ITALY 